\title{
ÉTUDE PHYSICO-CHIMIQUE DU LAIT
}

\author{
par
}

\section{W. KOPACZEWSKI}

\section{STABILISATION DU LAIT}

\section{Etat du problème}

Le problème de la stabilisation du lait, c'est-à-dire la possibilité, sinon de supprimer la gélification, tout au moins de la retarder considérablement, est d'une grande importance au point de vue alimentaire et au point de vue hygiénique.

La gélification du lait étant fonction de la température, le lait se gélifie très rapidement en été, et, dans les villes, à peine livré, ne supporte plus une ébullition. La pullulation des microorganismes est, en effet, en été rapide ; le lait, étant un milieu extrêmement propice pour eette pullulation, il se forme rapidement un mélangé de diverses cultures microbiennes, parmi lesquelles en dehors de nombreuses varitétés des microbes lactiques, que l'on peut considérer comme utiles à l'organisme en tant qu'antagonistes de certains microbes pathogènes, il $y$ en a qui peuvent être éminemment pathogènes et poussent en présence des bactéries lactiques.

Ces faits ont amené les producteurs à préparer des laits condensés ou secs; sans examiner ici les différences entre la valeur alimentaire de ces laits, notons que les laits condensés non sucrés ou insuffisamment sucrés ne se conservent pas une fois leurs récipients débouchés surtout pendant la période des chaleurs. Le problème n'est donc pas de cette façon entièrement résolu.

Pour toutes ces raisons l'industrie laitière est très intéressée par le problème de la stabilisation du lait et de ses dérivés tels que le beurre, la crème, etc. Divers stabilisants ont été proposés, lesquels retardent parfois assez nettement la gélification du lait à la température ambiante, mais un stabilisant idéal, permettant de faire figer pour ainsi dire l'état d'équilibre du lait, sans aucun inconvénient pour l'organisme, n'est pas encore trouvé.

On peut diviser les méthodes actuellement connues en physiques et chimiques.

Parmi les méthodes physiques rappelons, tout d'abord, les écarts de la température. Le lait refroidi à quelques degrés au-dessous de zéro peut être conservé durant plusieurs jours, et même semaines, sans altération. Ce procédé est parfait et sera de plus en plus fréquemment applicable sur le plan domestique ; quant au plan industriel, il nécessite le transport dans des voitures réfrigérées isothermes, ce qui grève le prix de revient de cet aliment. 
On a reproçhé à ce procédé le défaut d'homogénéité du lait décongelé ; on a donc proposé la «semi-congélation " : elle consiste à refroidir à $-120 \mathrm{C}$. un quart de lait à conserver et de mélanger ce bloc avec le reste; la température du lait s'établit alors vers $-4^{\circ} \mathrm{C}$. sous l'influence du volant de froid ainsi obtenu.

L'élévation de la température ne donne pas de résultats aussi satisfaisants. La chauffe durant une demi-heure à $62-85^{\circ} \mathrm{C}$., ou celle durant une demi-minute à $70-72^{\circ} \mathrm{C}$. ("pasteurisation " « basse " ou " haute "), ne détruit pas tous les microbes, et surtout laisse intactes, les espèces sporulées, de sorte que la gélification du lait s'ensuit avec un léger retard seulement, par rapport au lait non "pasteurisé ". On a essayé d'améliorer ces deux procédés en chauffant le lait à $75^{\circ}$ C., pendant quelques secondes, entre deux plaques métalliques peu éloignées l'une de l'autre; l'opération s'effectue dans des récipients clos, à l'abri des phénomènes d'oxydation, le lait s'écoulant en nappe mince entre les deux plaques. Cette manière de faire est utilisée en Italie sous le nom de "stassanisation " ; certains prétendent qu'elle aboutit à la destruction de la flore pathogène du lait et à la conservation de sa eomposition physico-chimique " $(\mathrm{KAY})$. En ce qui concerne cette dernière affirmation conservation des caractères physico-chimiques du lait - elle semble inexacte : en effet, le lait chauffé au voisinage de $60^{\circ} \mathrm{C}$. n'a pas les mêmes caractères physico-chimiques que le lait non chauffé : sa tension superficielle est augmentée (DAHLBERG, Hening, Joignerez, Cardoso et WAUColle; Kopaczewski, et autres) ; sa viscosité est plus accentuée (TAYLOR, KOPACZEWSKI et autres) ; sa concentration ionique globale plus basse (KoPAczEWSKI) ; ainsi que son degré d'hydratation micellaire (KoPACzeWSKI) ; enfin, son degré de dispersion diminue (LEMANISSIER) et le taux d'ionisation des sels calciques est abaissé à 40\% environ (Grosser). On peut conclure que la chauffe du lait dans les conditions réalisées à ce jour modifie l'équilibre colloïdal de ce système.

Il convient aussi d'attirer l'attention sur la nécessité de refroidir rapidement le lait pasteurisé car, sans cette précaution il reste un temps trop prolongé dans une zone de température favorable à la multiplication des microbes $\left(35-40^{\circ}\right.$ C.) survivant après la chauffe.

Enfin, la pasteurisation, plus encore que la réfrigération, augmente le prix de revient du lait. Pour cette raison, entre autres, on ne livre pas au commerce du lait bouilli ou stérilisé qu'exceptionnellement, notamment pour l'usage thérapeutique. On sait que seule la stérilisation normale - chauffe dans des vapeurs d'eau (autoclave) durant 20 minutes à $115^{\circ} \mathrm{C}$. permet la destruction totale des microbes sporulés. Mais, un tel lait acquiert un goût 
caramélisé, et une odeur particulière, rance, due à la saponification et à l'oxydation des lipides, ainsi qu'une couleur foncée jaunâtre.

En poussant plus longtemps la durée de la chauffe on obtient des laits condensés sucrés ou non sucrés, ainsi que des laits sees. On doit se rappeler que la préparation de la confiture de lait est depuis-de très longue date pratiquée en Amérique latine. Il va sans dire que tous ces laits industriels n'ont pas la même valeur alimentaire que le lait frais, pur, complet, malgré de réels progrès réalisés récemment, notamment dans la fabrication des laits sees instantanément dispersibles dans de l'eau et reprenant la saveur, la couleur et l'odeur du lait frais ; toutefois, dans ce cas aussi, les caractères physicochimiques du lait sont modifiés, son équilibre colloïdal altéré, sinon rompu. Nous avons déjà donné plusieurs exemples de ces altérations, mais c'est un problème intéressant au point de vue de l'hygiène et il est en dehors du plan de cet ouvrage.

Parmi les autres agents physiques utilisés comme stabilisants, examinons les rayons ultra-violets. En 1877 déjà, Downes et BLINT utilisaient des rayons de $280 \mathrm{~m} \mu$ de longueur d'onde pour stériliser le lait; Courmont et Nogier, en 1909, ont vu que l'action des rayons courts est plus efficace, notamment celle d'une longueur de $225 \mathrm{~m} \mu$. STOUTz a avancé que ces rayons détruisent les microbes pathogènes et laissent intactes les variétés lactiques. Soulignons que sous l'influence de ces radiations les stérols se transforment en vitamine D ; ce côté de la question présente un intérêt physiologique indéniable souligné par LASABLIk̀RE. Toutefois, d'après BLANCHARD, le lait irradié perd $10 \%$ de vitamine B2, $16 \%$ - de la vitamine B11 et environ $15 \%$ - de la vitamine C. Cette question fut examinée en 1937 par VIELLY et HARDER.

Les rayons infra rouges, par leurs effets thermogènes ont été également appliqués à la stabilisation du lait, mais la pratique de cette action n'a pas donné de résultats encourageants.

Enfin, on a voulu également utiliser les ultra-sons en " homogénéisant " et, par leur effet thermique, en stabilisant le lait ; mais, l'effet thermique des ultra-sons dépend de la nature du milieu et il n'est pas très accentué : $2 \mathrm{~cm}^{3}$ 'd'eau distillée placés dans un tube en verre s'échauffent de $2^{\circ} \mathrm{C}$. en dix secondes, tandis que la glycérine est rechauffée de $10^{\circ} \mathrm{C}$., tout comme l'huile de paraffine ; l'hydrosol de gélose ne s'échauffe que d'un degré à peine. Il se peut que le choix approprié d'appareillage permettra d'utiliser cette méthode, trop récente pour avoir dit son dernier mot.

Demeter, en 1941, a signalé que les ultra-sons possèdent une action bactéricide pour Bac. coli, Bac. typhi et Bac. dysenteriae; ce pouvoir serait plus faible envers les autres bactéries du lait. 
Parmi les agents physiques on a appliqué l'action des courants électriques variés et sous formes variées pour stabiliser le lait.

Gratz, en 1934, a proposé l'électrolyse par un courant continu et la désacidification consécutive; PIEN, puis DENIER, ont repris l'étude de ce procédé.

Les ondes courtes furent proposées en 1911 par Lewis : sous 2.500 à 6.000 volts de tension, le lait s'échauffe à $62-64^{\circ} \mathrm{C}$. Notons que Bessemans et Van MeirhaEde ont signalé l'effet antibiotique des ondes courtes ; d'après GrLLE, il serait dû à un " effet thermique électif ».

Enfin, en 1935, YaLE a décrit un procédé électrique de stabilisation du lait. On trouvera les détails sur ces procédés dans le travail de Decate.

Tel est l'ensemble des procédés proposés pour stabiliser le lait par les agents physiques.

Les agents chimiques ont été utilisés empiriquement depuis des temps immémoriaux sous formes variées : le vin, l'eau-de-vie, le vinaigre, la fumée des feuilles, etc. Puis, on a proposé de très nombreuses substances chimiquement définies; telles que les acides variés, les aldéhydes, les antiseptiques, la gélatine, la dextrine, le gaïacol, etc. L'addition de la gélatine fut préconisée par JACOBI en 1879 et l'idée a été reprise par ALEXANDER et appliquée à l'alimentation des enfants.

Voici les principaux stabilisants proposés :

TABLEAU XXXVIII

PRINGIPAUX STABILISANTS

\begin{tabular}{|c|c|c|}
\hline Substances & Concentrations $(\%)$ & Auteurs \\
\hline 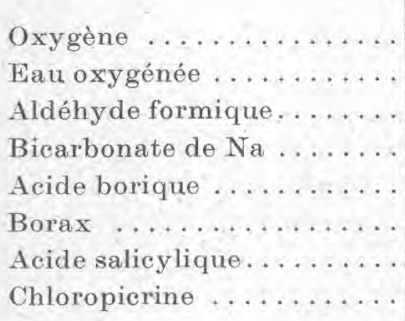 & $\begin{array}{l}\quad \text { pure } \\
2,0-3,0 \\
0,02 \\
0,1 \\
0,2 \\
0,02-0,5 \\
0,01 \\
0,01\end{array}$ & $\begin{array}{l}\text { Hofius } \\
\text { Bert et Regnard, } 1882 \\
\text { Trillat, } 1904 \\
\text { Bertrand (G), } 1944\end{array}$ \\
\hline
\end{tabular}

Pour compléter cette énumération, nous avons signalé les propriétés antigélifiantes d'une plante marocaine de la famille des composés "Senecio anteuphorbium", mais ce pouvoir est faible, et de plus, dépend de la concentration utilisée : en concentration de 
$2,5 \%$ - il retarde la gélification du lait frais de 2.3 heures; en eelle de $10 \%$ - de 6 heures à $25 \%$ - il est sans effet.

Notons que la plupart de ces substances antigélifiantes ont été utilisées pour la conservation du beurre, de la crème, de jus de fruits, de conserves variées, etc.

L'usage de ces substances est actuellement autorisé dans divers pays, sauf en France, où la loi interdit l'addition de toutesubstance chimique de synthèse aux produits alimentaires, eaux minérales, etc. Cette loi est l'expression d'une grande sagesse et d'une circonspection louable, car on ne peut affirmer, sans longues et systématiques expériences, qu'une substance déterminée soit sans un effet nocif, à la longue, pour l'organisme humain, qu'elle n'affaiblit pas les qualités nutritives d'un aliment, ou bien, qu'elle ne détruit pas l'équilibre minéro-minéral ou minéro-organique des humeurs; les récentes investigations sur les vitamines et sur la composition minérale concomitante des aliments ont attiré l'attention des physiologistes sur ce sujet. Toutefois, divers accommodements et tolérances se sont faits jour dans la législation française : on autorise l'addition des substances d'origine vitale - végétale ou animale. Ainsi, l'acide carbonique provenant d'une source minérale peut être introduit pour stabiliser une eau minérale, mais non celui obtenu par synthèse; la coloration du beurre par les colorants isolés des carottes est tolérée, mais pas celle à l'aide des colorants de synthèse. On peut admettre cette tolérance, mais il n'en est pas de même lorsqu'il s'agit d'une récente autorisation, cette autorisation d'ailleurs temporaire $a$, depuis, été rapportée, donnée par l'Académie de Médecine et par le Conseil supérieur d'Hygiène concernant l'addition au lait de la chloropicrine, gaz de combat dont la toxicité est égale à celle de l'acide eyanhydrique. On peut done souscrire à l'opinion de THIEULIN selon lequel "l'emploi officiel d'un antiseptique aurait pour effet de perpétuer et d'encourager une production défectueuse, de tromper le consommateur, et de nuire au progrès réel que les hygiénistes réclament et que de nombreux industriels poursuivent ". MARTEL a répété que " des produits en apparence inoffensifs peuvent devenir dangereux à la longue. ")

C'est done dans la voie des recherches d'un produit biologique normalement présent dans le règne végétal ou animal que devrait s'engager l'investigation concernant la stabilisation du lait.

Examinons donc les résultats des recherches concernant la stabilisation du lait obtenue dans divers pays où l'addition des stabilisants chimiques est autorisée (Allemagne, Danemark, Italie, Etats-Unis du Nord d'Amérique, ete.).

En ce qui concerne l'eau oxygénée, notons qu'elle agit par son oxygène : en effet, sous l'action de la catalase du lait ce produit est rapidement dédoublé en eau et en oxygène in statu nascendi. On a 
vite constaté que sous pression normale ce gaz n'agit que faiblement et, du reste, s'échappe rapidement ; en l'utilisant sous pression modérée et à la température de $75^{\circ} \mathrm{C}$. on peut vraiment stériliser le lait. Toutefois, on a signalé que la consommation des laits ainsi traités provoque chez les enfants une recrudescence de la maladie de Barlow.

L'addition de l'eau oxygénée en concentration finale de 2 à $3 \%$ retarde de 2 à 3 jours la gélification du lait, mais n'arrête la croissance des microorganismes que de quelques heures à peine (NICoLLE et DUClaUX). Il est encore à vérifier que cette addition soit sans action sur l'équilibre physicochimique du lait malgré l'affirmation de Monvoisin.

On.a donné la préférence, à notre époque, à l'action directe de l'oxygène sous pression. Notons que, déjà en 1884, Chauveau a démontré l'action bactéricide de l'oxygène sous pression ; c'est en se souvenant de cette constatation que le premier brevet américain fut pris en 1911 ; de nombreux autres publiés depuis diffèrent par de menus détails. D'après Gorini, on soumet le lait à une température de $55^{\circ} \mathrm{C}$. et à l'action de l'oxygène sous pression de 10 atmosphères durant 5 heures ; ce procédé fut appliqué avec de bons résultats en Italie en 1940-1944. Mais, on a signalé depuis que cette action de l'oxygène détruit partiellement les vitamines $\mathrm{C}$ et $\mathrm{A}$ (VAOHER).

Enfin l'ozonisation du lait fut pratiquée ; on sait que l'ozone a des propriétés bactéricides assez puissantes (FRoEHLICH) ; malheureusement, par ses propriétés oxydantes énergiques, il oxyde les graisses et communique au lait un goût rance particulier.

L'aldéhyde formique est un agent stabilisant actif. Notons tout d'abord que le produit commercial n'est pas une simple solution de ee gaz : il contient, en effet, $30-40,0 \%$ d'aldéhyde formique mais aussi $10-15,0 \%$ de méthanol, de traces d'acides, formique et acétique, ainsi que de produits de polymérisation de cetaldéhyde tels que paraformaldéhyde, aldéhyde formique hydraté, etc. Promoteur de cette méthode, Trillat souligne que l'aldéhyde formique persiste dans du lait ainsi traité même après l'ébullition, il peut done présenter des inconvénients par sa toxicité, quoique sa dose stabilisante est relativement faible. Nous reviendrons sur ce point dans la partie expérimentale. Il est, toutefois, établit par SHERMan que l'aldéhyde formique disparaît vingt fois plus rapidement du lait que de sa solution aqueuse de même concentration. On sait en outre, que l'aldéhyde formique est un réactif des protides qu'il les flocule (Merck, Fouassier, Nitschmann et Ladener, et autres).

Mais nous verrons que e'est une question de concentration.

On a signalé, enfin, que l'acide formique et ses sels ne semblent 
pas avoir d'effets toxiques en concentration au-dessous de $0,65 \%$ (KREIL).

ZUNZ a soutenu que l'emploi de l'acide borique et des borates est contre-indiqué, étant donné qu'ils "empêchent la coagulation de la caséine "; c'est un argument surprenant, car, précisément, on cherche à empêcher cette gélification dans du lait.

Examinons le,cas de la chloropicrine (microlysine). Il s'agit en l'occurence d'une substance éminemment toxique ; on sait que e'est un "gaz de combat". C'est une substance liquide dont le point d'ébullition est à $112^{\circ} \mathrm{C}$. ; sa solubilité est faible $\left(2,4 \% \circ\right.$ à $20^{\circ} \mathrm{C}$. dans l'ean distillée d'après PIEN). Voici sa toxicité, comparée aux autres "gaz de guerre": la mort s'ensuit en une minute pour l'homme, d'après Meyer, en concentration de : "

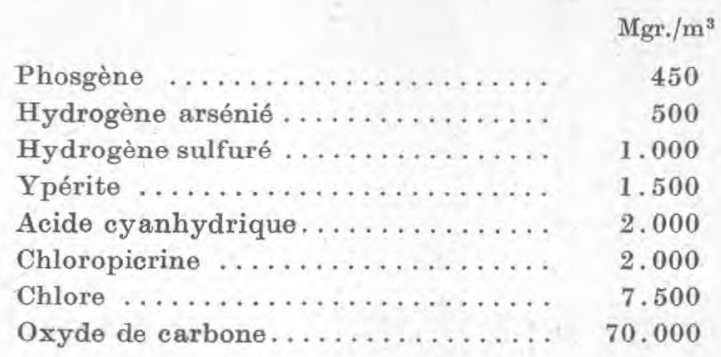

A des doses infimes, la chlopicrine provoque une irritation des voies respiratoires, se traduisant par le picottement et la sensation de cuisson dans la gorge, la tête sèche, etc. Ce sont, précisément, ces symptômes que l'on a signalé à la suite d'ingestion du lait additionné de 0 gr. 080 par litre de chloropicrine (PIEN). Du reste, au point de vue purement physicochimique le lait additionné de la dose en question ne se gélifie qu'avec un retard de 4 jours, et, au point de vue bactériologique, de tels laits sont aussi pollués que le lait non traité (PIEN). L'affirmation de G. BERTraND, selon laquelle l'ébullition du lait additionné de chloropicrine permettrait de chasser cette substance, semble, a priori problématique, étant donné le point de l'ébullition relativement élevée de cette substance ; elle n'est pas confirmée par l'expérience. Du reste, plusieurs faits d'observation courante montrent que certaines substances volatiles sont très difficiles à éliminer de leurs mélanges avec du lait. Ainsi, nous avons $v u$ que l'aldéhyde formique pratiquement ne peut pas être éliminée (Trillat) : on sait qu'il en, est de même pour l'éther éthylique mélangé avec de l'huile, même au bout de plusieurs heures de chauffe; nous avons constaté que l'on n'arrive point à chasser de la même façon l'éther acétique du lait. 
De plus, au point de vue physicochimique, le lait ainsi traité flocule par l'ébullition (PIEN).

Nous n'insisterons pas sur la pratique de stabilisation concernant le sublimé employé aux Etats-Unis d'Amérique du Nord pour "stabiliser " la crème glacée, ni sur l'utilité, douteuse, d'incorporation de bromo-acétate d'éthyle, etc.

Terminons cet exposé en signalant les recherches récentes concernant la toxicité de quelques autres substances préconisées comme stabilisantes du lait, telles que propionate de $\mathrm{Ca}$ ou de $\mathrm{Na}$, le chlorure d'alcoyl-di méthyl-benzyl-ammonium ("zéphiran »), le benzoate de sodium (HARASHBARHER), acide $\beta$-oxy-benzoïque (" nipagine $)$, etc.

Mais, il va sans dire que toutes ces substances sont très intéressantes à étudier au point de vue théorique : en effet, croyons-nous, c'est en multipliant ces recherches que l'on arrivera, peut-être, à accumuler une série de faits expérimentaux dont la comparaison avec leurs caractères physiques et chimiques permettra d'entrevoir le mécanisme de leur action et, ipso facto, de découvrir, plus facilement et non empiriquement, des stabilisants pratiquement utilisables.

C'est dans cette voie que nous nous sommes engagés en tirant profit des résultats de nos recherches concernant la stabilisation des colloïdes en général, ainsi que du latex et du sang, en particulier.

\section{B. Partie expérimentale}

Nous avons publié en 1943 les premiers résultats de nos recherches; nous les avons élargies depuis sur d'autres substances ; de plus, nous les avons contrôlées sur du lait de France moins souillé que le lait marocain ; enfin, en dehors du lait frais complet nous avons utilisé le lait concentré, sucré ou non sucré, et le lait sec.

Ces recherches ont été effectuées dans les conditions suivantes : le lait fut additionné de divers stabilisants en concentrations variées et, une fois l'homogénéité du mélange assuré par l'agitation, on laissait les tubes contenant les mélanges en question à la température du laboratoire (15-20 C.) sans les boucher. Afin de pouvoir tirer quelques conclusions valables sur le mécanisme de la stabilisation du lait, nous ne nous sommes pas contenté d'examiner la gélification, sous l'influence des acides produits par les microorganismes, mais aussi, en éliminant ces derniers, celle produite par l'acide lactique, et, enfin, par la présure. C'est en comparant ces résultats que nous pourrons savoir quel est le véritable mécanisme de la stabilisation, ou, tout au moins, de l'entrevoir. La recherche des stabilisants corrects pourra ainsi être facilitée. 


\section{Stabilisation du lait au contact des microorganismes}

a) Stabilisants chimiques connus. Donnons, tout d'abord, les résultats concernant les stabilisants déjà utilisés :

TABLEAU XXXIX

STABILISANTS CONNUS ET LA GÉLIFICATION DU LAIT (A $15^{\circ}$ C.)

\begin{tabular}{|c|c|c|}
\hline Substanees & $\begin{array}{c}\text { Concentrations } \\
\text { finales }(\%)\end{array}$ & $\begin{array}{l}\text { Retard de la } \\
\text { gélification (jours) }\end{array}$ \\
\hline \multirow{3}{*}{ Acide salicylique....... } & 0,001 & 0 \\
\hline & 0,002 & 0,2 \\
\hline & $0,005 \cdot 0,01$ & 0 \\
\hline \multirow[t]{3}{*}{ Acide borique . } & 0,126 & 0,2 \\
\hline & 0,242 & 1,0 \\
\hline & 0,52 à 0,9 & 0 \\
\hline \multirow{4}{*}{$\mathrm{H}_{2} \mathrm{O}_{2}$. } & 0,24 (en volume) & 0 \\
\hline & 0,48 & 2,0 \\
\hline & 0,95 & 3,5 \\
\hline & 1,80 & 5,0 \\
\hline \multirow[t]{5}{*}{$\mathrm{NaHCO}_{3}$} & 0,30 & 1,0 \\
\hline & 0,60 & 2,5 \\
\hline & 1,20 & 3,0 \\
\hline & 2,20 & 5,0 \\
\hline & 4,00 & Floculation \\
\hline \multirow{2}{*}{$\mathrm{Na}_{3} \mathrm{BO}_{3}$} & $0,02-0,04$ & 0 \\
\hline & 0,08 & 1,0 \\
\hline \multirow[t]{3}{*}{$\mathrm{K}_{2} \mathrm{Cr}_{2} \mathrm{O}_{4}$} & 0,065 & Floculation \\
\hline & 0,125 & 20,0 \\
\hline & $0,05-0,24$ & 23,0 \\
\hline \multirow{9}{*}{$\begin{array}{l}\text { Aldéhy de formique...... } \\
\text { (Commerce à } 40 \% \text { ) }\end{array}$} & 0,06 & 1,0 \\
\hline & 0,08 & 2,5 \\
\hline & 0,10 & 2,5 \\
\hline & 0,12 & 4,0 \\
\hline & 0,14 & 6,5 \\
\hline & 0,16 & 7,0 \\
\hline & 0,18 & 9,0 \\
\hline & 0,20 & 11,0 \\
\hline & 1,0 à $4,0 \%$ & Coagulation instantanée \\
\hline
\end{tabular}

L'examen de ce tableau permet de conclure que seuls le bicarbonate et, surtout, le bichromate et l'aldéhyde formique permettent une conservation assez prolongée du lait. A propos du dernier, il convient de signáler que, selon la dose, la gélification s'ácompagne 
TABLEAU XL STABILISANTS DU LAIT AVEG EFFET SECONDAIRE

\begin{tabular}{|c|c|c|c|}
\hline Substances & $\begin{array}{l}\text { Concentrations } \\
\text { finales }(\%)\end{array}$ & $\begin{array}{l}\text { Retard de la gelli- } \\
\text { fication (jours) }\end{array}$ & $\begin{array}{l}\text { Effets } \\
\text { secondaires }\end{array}$ \\
\hline Ammonium, fluorure . . . . . & 0,35 & 6,0 & Floculation \\
\hline Ammonim, sulfocyanure (1)... & 1,1 & 6,0 & Clarification \\
\hline Ammonium, oxalate ....... & 5,12 & 1,0 & Floculation \\
\hline Potassium, eyanure ....... & 0,56 & 3,0 & Clarification \\
\hline \multirow[t]{4}{*}{ Potassium, permanganate ... } & 0,5 & 0 & Floculation \\
\hline & 0,75 & 5,0 & $"$ \\
\hline & 1,0 & 1,0 & " \\
\hline & 1,25 & 6,0 & $n$ \\
\hline \multirow{4}{*}{ Sodium, bisulfite ........ } & 0,66 & 6,0 & Clarification \\
\hline & 1,32 & 8,0 & n) \\
\hline & 2,0 & 9,0 & $n$ \\
\hline & 4,0 & 13,0 & $n$ \\
\hline \multirow[t]{2}{*}{ Sodium, iodate $\ldots \ldots \ldots \ldots$} & 0,08 & 0 & - - \\
\hline & 0,16 & 10,0 & Floculation \\
\hline \multirow[t]{2}{*}{ Sodium, phosphate bisodique } & 0,84 & 4,0 & Clarification \\
\hline & 1,68 & 3,0 & 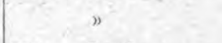 \\
\hline \multirow[t]{4}{*}{ Sodium, phosphate trisodique } & 0,15 & 2,0 & Floculation \\
\hline & 0,29 & 5,0 & 》 \\
\hline & 0,55 & 4,0 & $"$ \\
\hline & 1,03 & - & Coagulation \\
\hline Sodium, eitrate .......... & 0,96 & 8,0 & Clarification \\
\hline \multirow{3}{*}{ Magnésium, chlorure ...... } & 0,76 & 0 & Floculation \\
\hline & 1,50 & 5,0 & $n$ \\
\hline & 2,80 & 5,0 & 》 \\
\hline \multirow[t]{2}{*}{ Plomb, acétate } & 0,42 & 2,0 & Floculation \\
\hline & 0,63 & 6,0 & $n$ \\
\hline \multirow[t]{3}{*}{ Tri-ethanol-amine } & 2,0 & 0 & - \\
\hline & 4,0 & 3,0 & Clarification \\
\hline & $5,0 \quad(p \mathrm{H}=5,8)$ & 5,0 & 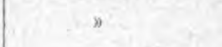 \\
\hline Di-chlore-éthane ......... & $2,0 \quad(p \mathrm{H}=4,8)$ & 10,0 & () \\
\hline \multirow[t]{4}{*}{ Tri-chlore-éthylène . . . . . . . } & 2,0 & 0 & ") \\
\hline & 4,0 & 3,0 & $\eta$ \\
\hline & 6,0 & 7,0 & $\Rightarrow$ \\
\hline & 10,0 & 19,0 & $n$ \\
\hline \multirow{4}{*}{ Tétrachlorure de carbone .... } & 2,0 & 0 & Clarification \\
\hline & 6,0 & 1,0 & n) \\
\hline & 8,0 & 7,0 & n \\
\hline & 10,0 & 17,0 & $n$ \\
\hline \multirow[t]{3}{*}{ Sulfure de carbone } & 2,0 & 50 & $+n$ \\
\hline & 4,0 & 9,0 & ) \\
\hline & 6,0 & 75,0 & $n$ \\
\hline Furfurol ............... & 2,0 & 15,0 & Floculation \\
\hline Nicotine .............. & 8,0 & $\begin{array}{l}\text { au delà de } \\
10 \text { jours }\end{array}$ & $\begin{array}{l}\text { Clarification } \\
\text { et floculatio }\end{array}$ \\
\hline
\end{tabular}

(1) L'action stabilisante de ce produit a été signalée en 1895 par Wroblewski. 
d'une synérèse parfois extrêmement abondante : ainsi pour les concentrations finales de $1,0 \%$ la synérèse est énorme, diminue avec celle de $1,2 \%$ et disparaît avec $1,6 \%$.

Le lait conservé par le formol peut être bouilli sans se gélifier, lorsqu'il est stabilisé par la concentration de $1,5 \%$ et conservé durant 5 jours à $320 \mathrm{C}$.

Notons, enfin, qu'avec des concentrations allant de $1 / 4.000$ à 1/8.000 on assiste à la formation d'un bouchon spumeux à la surface, le reste étant un liquide presque transparent.

Les concentrations responsables de ces phénomènes varient d'un lait à l'autre, et, par conséquent, nous les donnons à titre purement indicatif.

b) Stabilisants nouveaux à effets secondaires. Les stabilisants nouveaux que nous avons découverts peuvent être divisés en deux groupes : sans effets secondaires et possédant une action collatérale. A vrai dire, tout cela est une question de concentration : en effet, même l'aldéhyde formique, nous l'avons vu, dans une zone de concentrations déterminée, provoque des phénomènes collatéraux, tels que la clarification du lait, ou sa coagulation ; on peut, néanmoins, pour une concentration donnée, isoler dans certains cas un effet purement stabilisant. Toutefois, certaines substances, quelle que soit leur dose (dans nos conditions expérimentales cela va sans dire), tout en stabilisant le lait provoquent un effet secondaire tel que la floculation plus ou moins abondante ou la clarification du complex colloïdal. Voici les substances en question (tableau XL).

e) Stabilisants nouveaux sans effet secondaire apparent. Parmi les substances en question nous n'avons pas trouvé d'acides, mais des bases, des sels, des solvants, etc. Voici ces résultats (tableau XLI) concernant toutes les substances stabilisantes sans tenir compte de leurs effets secondaires variés.

TABLEAU XLI

STABILISANTS ENVERS LE LAIT

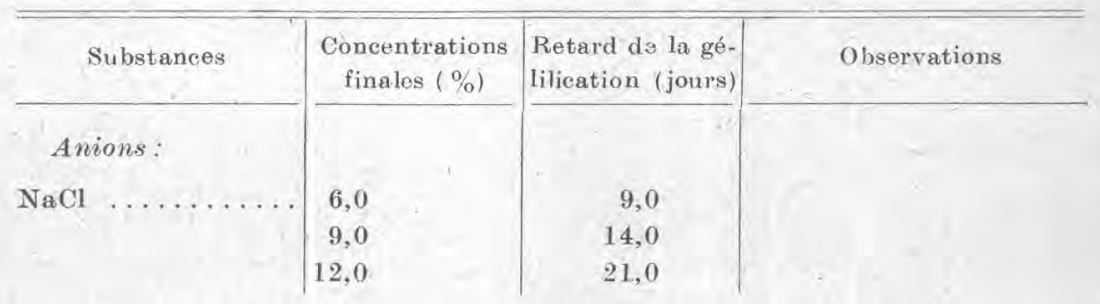


TABLEAU (suite)

\begin{tabular}{|c|c|c|c|}
\hline Substances & $\begin{array}{l}\text { Coneentrations } \\
\text { finales }(\%)\end{array}$ & $\begin{array}{l}\text { Retard de la gé- } \\
\text { lification (jours) }\end{array}$ & Observations \\
\hline \multirow[t]{3}{*}{$\mathrm{NaI}$} & 1,33 & 4,0 & , \\
\hline & 2,66 & 5,0 & \\
\hline & 5,32 & 7,0 & \\
\hline \multirow{3}{*}{$\mathrm{Na}_{2} \mathrm{~S}_{2} \mathrm{O}_{3}$} & $0,44-0,88$ & 6,0 & \\
\hline & 1,76 & 12,0 & \\
\hline & 2,20 & 17,0 & \\
\hline \multirow{2}{*}{$\mathrm{Na}_{2} \mathrm{SO}_{3}$} & 0,34 & 8,0 & \\
\hline & 0,68 & 15,0 & \\
\hline \multicolumn{4}{|l|}{ Cations: } \\
\hline \multirow{3}{*}{$\mathrm{AgNO}_{3}$} & 0,02 & 2,5 & \\
\hline & 0,04 & 3,0 & \\
\hline & 0,08 & 5,5 & \\
\hline \multirow[t]{2}{*}{$\mathrm{CaCl}_{2}$} & $1,3-2,6$ & 7,0 & \\
\hline & $5,2 \cdot 6,5$ & 10,0 & \\
\hline \multirow[t]{4}{*}{$\mathrm{CuCl}_{2}$} & 0,02 & 9,0 & \\
\hline & 0,04 & 11,0 & \\
\hline & 0,06 & 13,0 & \\
\hline & 0,08 & 15,0 & \\
\hline \multirow[t]{5}{*}{$\mathrm{CoCl}_{2}$} & 3,0 & 0,3 & \\
\hline & 6,0 & 1,0 & \\
\hline & 11,0 & 2,5 & \\
\hline & 20,0 & 3,0 & \\
\hline & 30,0 & 7,5 & \\
\hline \multirow[t]{3}{*}{$\mathrm{HgCl}_{2}$} & 0,02 & 4,0 & \\
\hline & 0,04 & 7,0 & \\
\hline & 0,08 & 30,0 & \\
\hline \multirow[t]{2}{*}{$\mathrm{PdCl}_{2}$} & 0,05 & 2,0 & \\
\hline & 0,10 & 6,5 & \\
\hline \multirow[t]{3}{*}{$\mathrm{FeCl}_{3}$} & 0,176 & 1,0 & \\
\hline & 0,258 & 2,0 & \\
\hline & 0,348 & 4,0 & \\
\hline \multicolumn{4}{|l|}{ Bases: } \\
\hline \multirow[t]{2}{*}{ Diméthylamine } & 1,0 & 1,0 & \\
\hline & 2,0 & 1,5 & \\
\hline \multirow[t]{2}{*}{$\bullet$} & 4,0 & 2,0 & \\
\hline & 8,0 & 6,0 & \\
\hline \multirow[t]{3}{*}{ Di-éthylamine } & $0,06-0,264$ & 0 & \\
\hline & 0,528 & 2,0 & \\
\hline & 1,056 & 4,0 & \\
\hline Chloramine $\mathrm{T}$ & 0,02 & 13,0 & \\
\hline
\end{tabular}


TABLEAU (suite)

\begin{tabular}{|c|c|c|c|}
\hline Substances - & $\begin{array}{l}\text { Concentrations } \\
\text { finales }(\%)\end{array}$ & $\begin{array}{l}\text { Retard de la gé- } \\
\text { lification (jours) }\end{array}$ & Observations \\
\hline Aniline .. & $1,0-2,0$ & 10,0 & \\
\hline - & 4,0 & 13,0 & \\
\hline \multirow[t]{2}{*}{ Pyridine .. } & 1,0 & 12,0 & \\
\hline & 2,0 & 30,0 & \\
\hline \multirow[t]{4}{*}{$\begin{array}{l}\text { Hexaméthylène- } \\
\text { tétramine } \ldots . . .\end{array}$} & & & \\
\hline & 1,75 & 21,0 & \\
\hline & 2,5 & 6,0 & \\
\hline & 5,0 & 13,0 & \\
\hline \multicolumn{4}{|l|}{ Solvants : } \\
\hline \multicolumn{4}{|l|}{ Ether de pétrole } \\
\hline \multirow[t]{3}{*}{ Benzène ... } & 2,0 & 1,0 & \\
\hline & 4,0 & 2,0 & \\
\hline & 8,0 & 11,0 & \\
\hline \multirow[t]{3}{*}{ Toluène. } & 2,0 & 1,0 & \\
\hline & 4,0 & 3,0 & \\
\hline & 8,0 & 14,0 & \\
\hline \multirow[t]{2}{*}{ Xylène } & 8,0 & 0,5 & \\
\hline & 16,0 & 15,0 & \\
\hline \multirow[t]{2}{*}{ Aleool éthylique } & 0,5 & 3,0 & \\
\hline & $1,0-16,0$ & 0 & Effets secondaires \\
\hline \multirow[t]{2}{*}{ Aleool iso-amylique } & 1,0 & 0,5 & \\
\hline & 2,0 & 15,0 & \\
\hline \multirow[t]{4}{*}{ Cyclohexanol...... } & 2,5 & 2,0 & \\
\hline & 5,0 & 3,0 & \\
\hline & 7,5 & 4,0 & \\
\hline & 10,0 & 5,5 & \\
\hline \multirow[t]{3}{*}{ Glyeol. } & 2,5 & 1,5 & \\
\hline & 5,0 & 2,5 & \\
\hline & 7,5 & 5,0 & \\
\hline \multirow[t]{2}{*}{ Di-éthylène-glycol } & $0,5 \cdot 5,0$ & 0 & \\
\hline & 15,0 & 8,0 & \\
\hline \multirow[t]{5}{*}{ Phénol } & 0,5 & 13,0 & \\
\hline & 1,0 & 16,0 & \\
\hline & 1,5 & 22,0 & \\
\hline & 2,0 & 16,0 & \\
\hline & 2,5 & 8,0 & \\
\hline \multirow[t]{3}{*}{ Ether éthylique.... } & 2,0 & 0 & \\
\hline & 4,0 & 0,5 & \\
\hline & 8,0 & 14,0 & Effets secondaires \\
\hline
\end{tabular}




\section{TABLEAU (fin)}

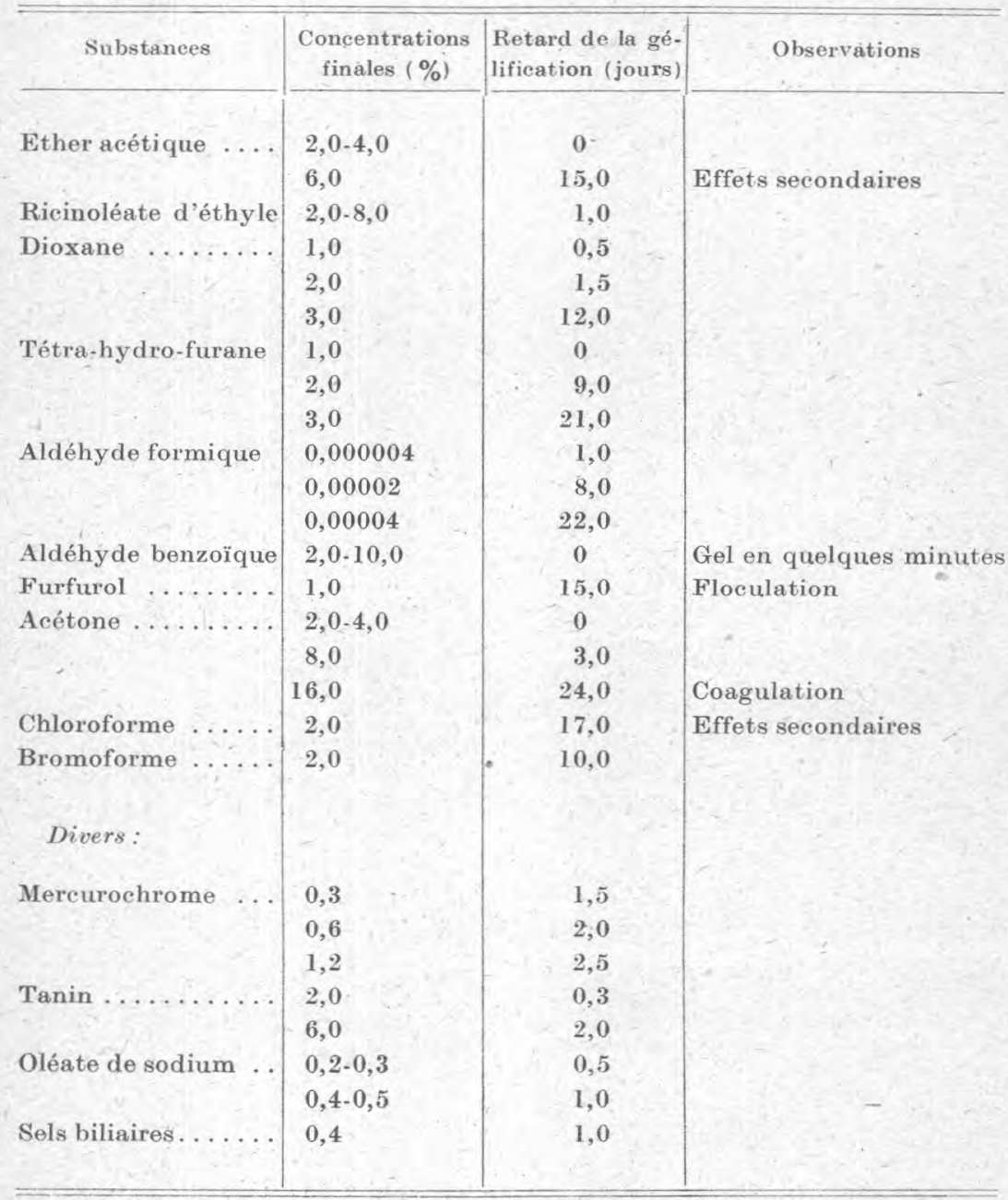

Bien d'autres substances empêchent la gélification du lait par les microorganismes, mais, en même temps, elles provoquent des effets secondaires variés que nous examinerons dans le chapitre suivant, en détails.

Enumérons les substances qui ne possèdent aucune action stabilisante, tout au moins dans nos conditions expérimentales, ou bien cette action est très faible ; voici cette liste accompagnée des concentrations expérimentées : 
TABLEAU XLII

SUBSTANGES SANS ACTION STABILISANTE

Substances

$\mathrm{NaNO}_{3} \ldots \ldots \ldots \ldots \ldots \ldots \ldots \ldots \ldots$

$\mathrm{NaBr} \ldots \ldots \ldots \ldots \ldots \ldots \ldots \ldots \ldots$

$\mathrm{PbCl}_{2} \ldots \ldots \ldots \ldots \ldots \ldots \ldots$

Glucose....................

Benzoate de benzyle $\ldots . . \ldots \ldots \ldots \ldots$

Essence de térébenthine ............

$\mathrm{NaNO}_{3} \ldots \ldots \ldots \ldots \ldots \ldots \ldots \ldots$

Acétate de $\mathrm{Pb} \ldots \ldots \ldots \ldots \ldots \ldots \ldots$

Chlorure de $\mathrm{Pb} \ldots \ldots \ldots \ldots \ldots \ldots \ldots$

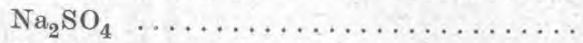

Ferricyanure de $\mathrm{Na} \ldots \ldots \ldots \ldots \ldots \ldots$

Ferrocyanure de $\mathrm{Na}$
Concentrations étudiées finales $(\%)$

$0,13-0,42$

$0,1-0,3$

$0,012-0,05$

$1,0 \quad-16,0$

$1,0 \quad-8,0$

$1,0 \quad-10,0$

$0,17-0,68$

$0,03-0,12$

$0,012 \cdot 0,06$

$0,08-0,32$

$0,12 \cdot 0,48$

$0,10 \cdot 0,40$

d) Observations générales. Ces données doivent être accompagnées de quelques observations.

Tout d'abord, la stabilisation est, en général, fonction de la concentration ; c'est un phénomène périodique. Parfois, ce sont les faibles doses qui stabilisent et les fortes détruisent l'équilibre colloïdal, en provoquant soit une floculation, soit une coagulation hétérogène, soit enfin, une décoloration totale. Ainsi, le bicarbonate de $\mathrm{Na}$, l'acétate de $\mathrm{Pb}$, l'iodate de $\mathrm{N}$, le chlorure de palladium et l'hexaméthylène tétramine provoquent la floculation à des doses fortes, tandis que te bichromate et le permanganate de potassium, le fluorure de sodium et le chlorure de magnésium floculent aux doses plus faibles que celles qui stabilisent: Cette floculation n'empêche pas toujours la gélification de s'ensuivre : c'est le cas, par exemple, du chlorure de palladium et du sulfocyanure d'ammonium. Dans les deux cas, on voit la formation d'un dépôt au fond des récipients.

Dans d'autres cas, c'est une coagulation hétérogène qui s'observe au bout d'un certain"temps, durant lequel le lait reste liquide : par exemple, avec le sulfite et l'iodate de sodium, etc. Parfois, cette coagulation fait place à une véritable gélification : ainsi les coagels d'acétate de plomb se transforment en gels au bout de 8 jours, gels compacts quoique fêlés.

Certains solvants provoquent la dissolution des graisses et la séparation de cette phase liquide (sulfure de carbone, dichloroethane, tri-chlore-éthylène, et tétrachlorure de carbone).

Notons que ces phénomènès n'empêchent pas la gélification ultérieure du lait, mais, dans ces cas, les gels sont transparents.

Ainsi, pour obtenir une stabilisation du lait, il faut fixer minutieusement la zone de concentrations : l'exemple des cations est 
particulièrement frappant à ce point de vue : ils gélifient parfois instantatément le lait à la concentration appropriée $(\mathrm{Ca}, \mathrm{Cu}, \mathrm{Fe}$, $\mathrm{Hg}$, etc.) et à des doses faibles ils stabilisent le système colloïdal en question. Nous reviendrons sur ce point en discutant le mécanisme d'action de toutes ces substances.

En dehors de la concentration, la stabilisation est fonction de la température: les doses stabilisantes que nous avons données sont valables pour les températures de $15-20^{\circ} \mathrm{C}$.; il n'en est pas de même pour la température plus élevée. Voici quelques exemples à ce sujet (tableau XLIII).

TABLEAU XLIII

STABILISATION ET LA TEMPÉRATURE

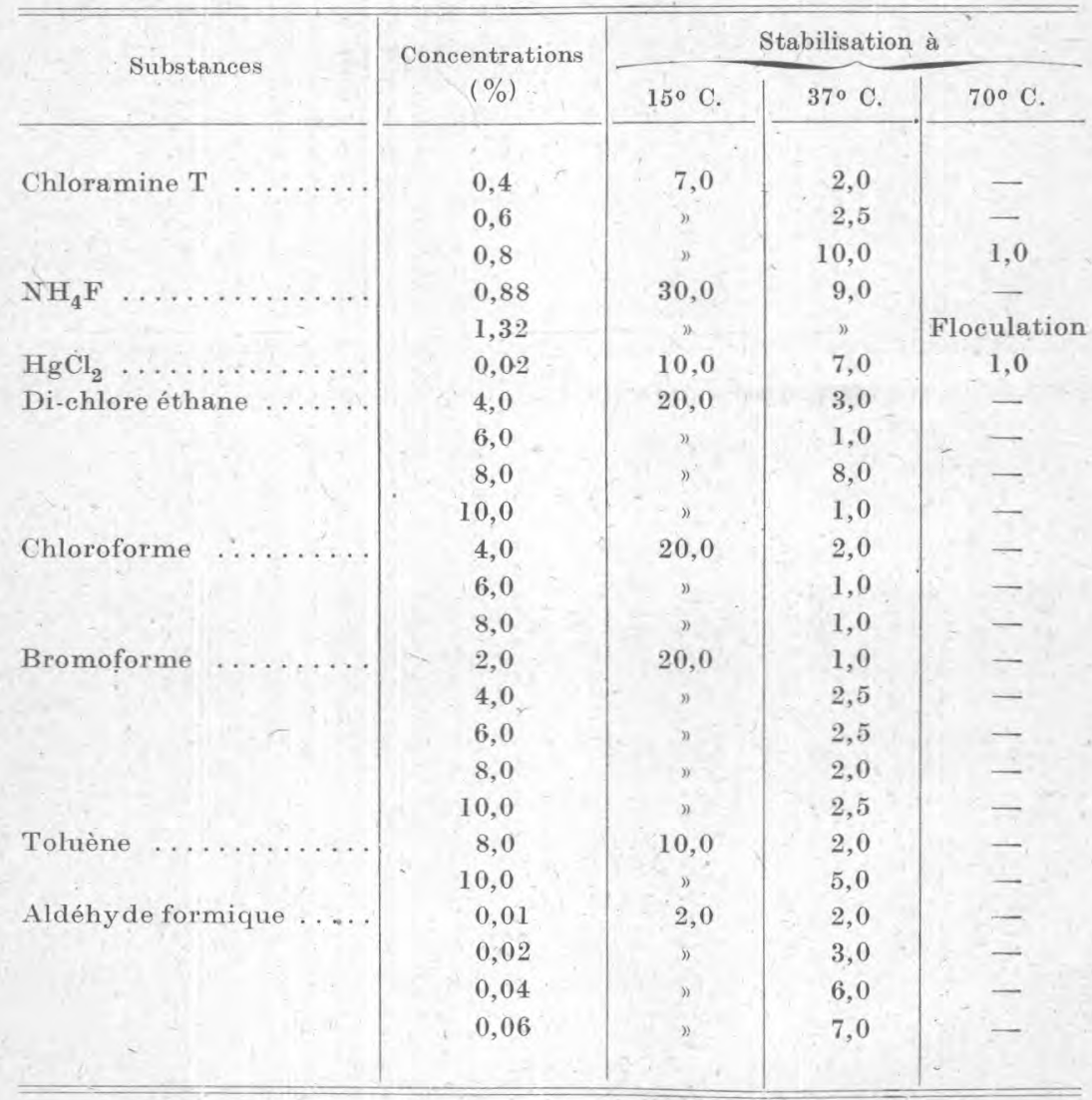

La stabilisation varie également d'un lait à l'autre, et les concentrations stabilisantes doivent subir des corrections parfois appréciables. Ces corrections s'accentuent lorsque le lait a subi des manipulations variées telles que la condensation, dessiccation et 
l'addition de sucre. Voici à ce sujet quelques chiffres (tableau XLIV).

TABLEAU XLIV

STABILISATION DES LAITS INDUSTRIELS

\begin{tabular}{|c|c|c|c|c|c|}
\hline \multirow{2}{*}{ Stabilisants } & \multirow{2}{*}{$\begin{array}{c}\text { Concentra- } \\
\text { tions } \\
\text { finales }(\%)\end{array}$} & \multicolumn{4}{|c|}{ Eehantillons de lait : } \\
\hline & & frais & bouilli & condensé & sec \\
\hline \multirow{2}{*}{$\mathrm{CuCl}_{2}$. } & 0,02 & 9,0 & 17,0 & 6,0 & 8,0 \\
\hline & 0,04 & 13,0 & 11,0 & 7,0 & 12,0 \\
\hline Témoin (gels en jours). & ' & 2,5 & 3,0 & 4,0 & 4,0 \\
\hline
\end{tabular}

Et voici les variations d'un lait frais à l'autre :

TABLEAU XLV

STABILISATION DU LAIT FRAIS PAR L'ALDÉHYDE FORMIQUF

\begin{tabular}{c|r|r|r|r|r}
\hline \hline $\begin{array}{c}\text { Concentra- } \\
\text { tions } \\
\text { finales (\%) }\end{array}$ & \multicolumn{5}{|c|}{-Gélification en jours } \\
\cline { 2 - 4 } & I & II & III & IV & V \\
\cline { 2 - 4 } 0,01 & 6,0 & 2,0 & 5,0 & 8,0 & 2,5 \\
0,04 & 14,0 & 8,0 & 22,0 & 12,0 & 15,0 \\
\hline \hline
\end{tabular}

Tels sont les faits et les observations générales concernant la stabilisation du lait. Etudions un peu l'éventuel mécanisme de cette stabilisation.

\section{Stabilisation du lait au contact de l'acide lactique}

Nous avons expérimenté la plupart des substances qui empêchent énergiquement la gélification du lait par l'acidification microbienne. Le mode expérimental était le suivant : le lait fut, tout d'abord, additionné de stabilisants variés, agité, pour effectuer une homogénéisation correcte, puis additionné d'acide lactique, pur Codex français, à raison de $1 \%$ en concentration finale (II gouttes pour $5 \mathrm{~cm}^{3} \mathrm{du}$ lait). Ces expériences ont été, en outre, accompagnées d'une épreuve concernant l'action de l'acide lactique sur les réactifs utilisés en solutions aqueuses. L'ensemble de ces données est récapitulé dans le tableau ci-après (tableau XLVI).

Ajoutons à cela que le bisulfite et le sulfite de $\mathrm{Na}$, ainsi que le cyanure de potassium semblent retarder d'environ 72 heures la gélification du lait par l'acide lactique en concentration finale de $1,0 \%$. 


\section{TABLEAU XLVI}

\section{STABILISATION DU LAIT SOUMIS A L'ACTION DE L'ACIDE LAGTIQUE}

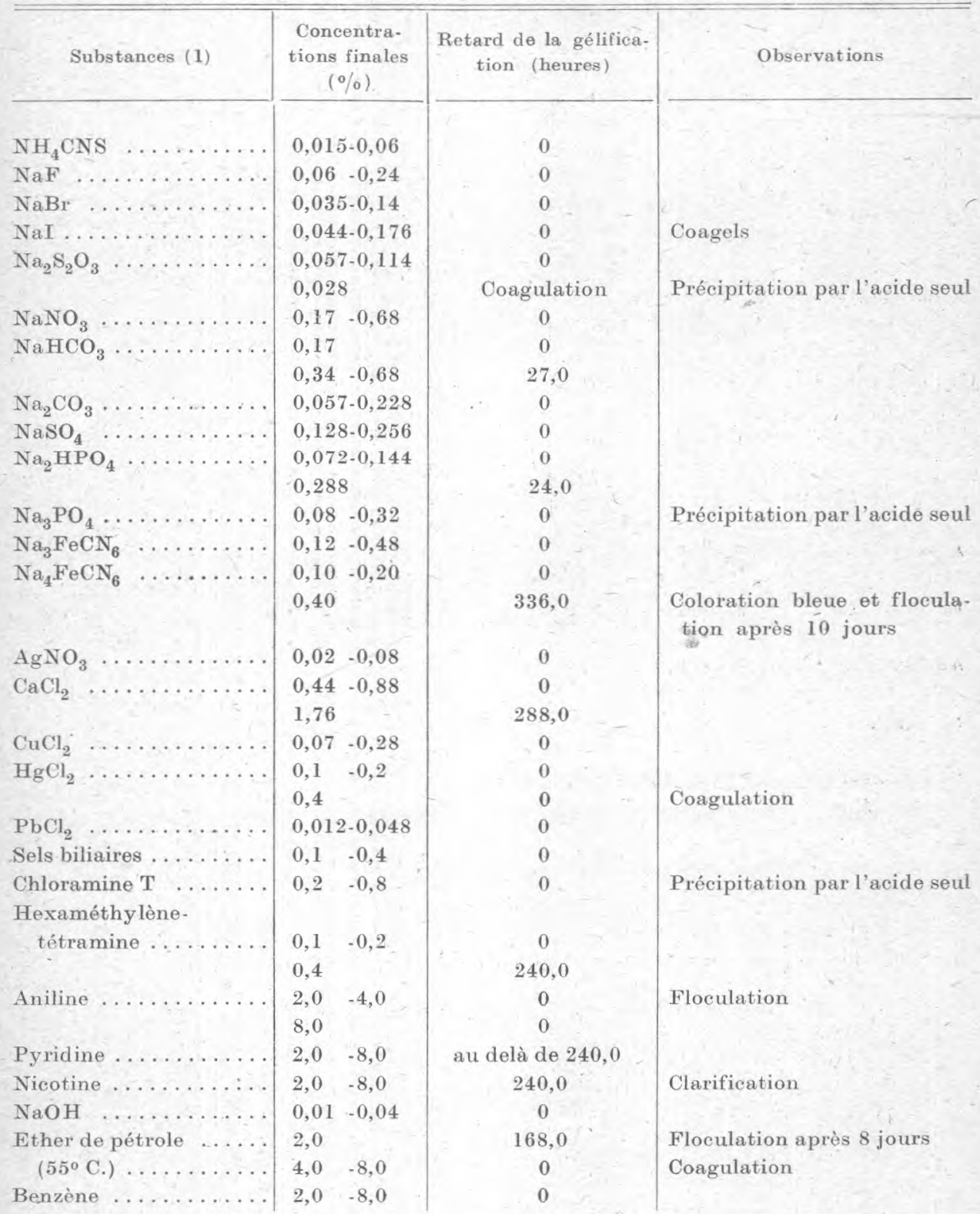

(1) On trouvera dans ce tableau plusieurs substanees dont l'effet stabilisant s'accompagne d'autres phénomènes secondaires, tels que clarification, floculation, etc. 
TABLEAU (suite)

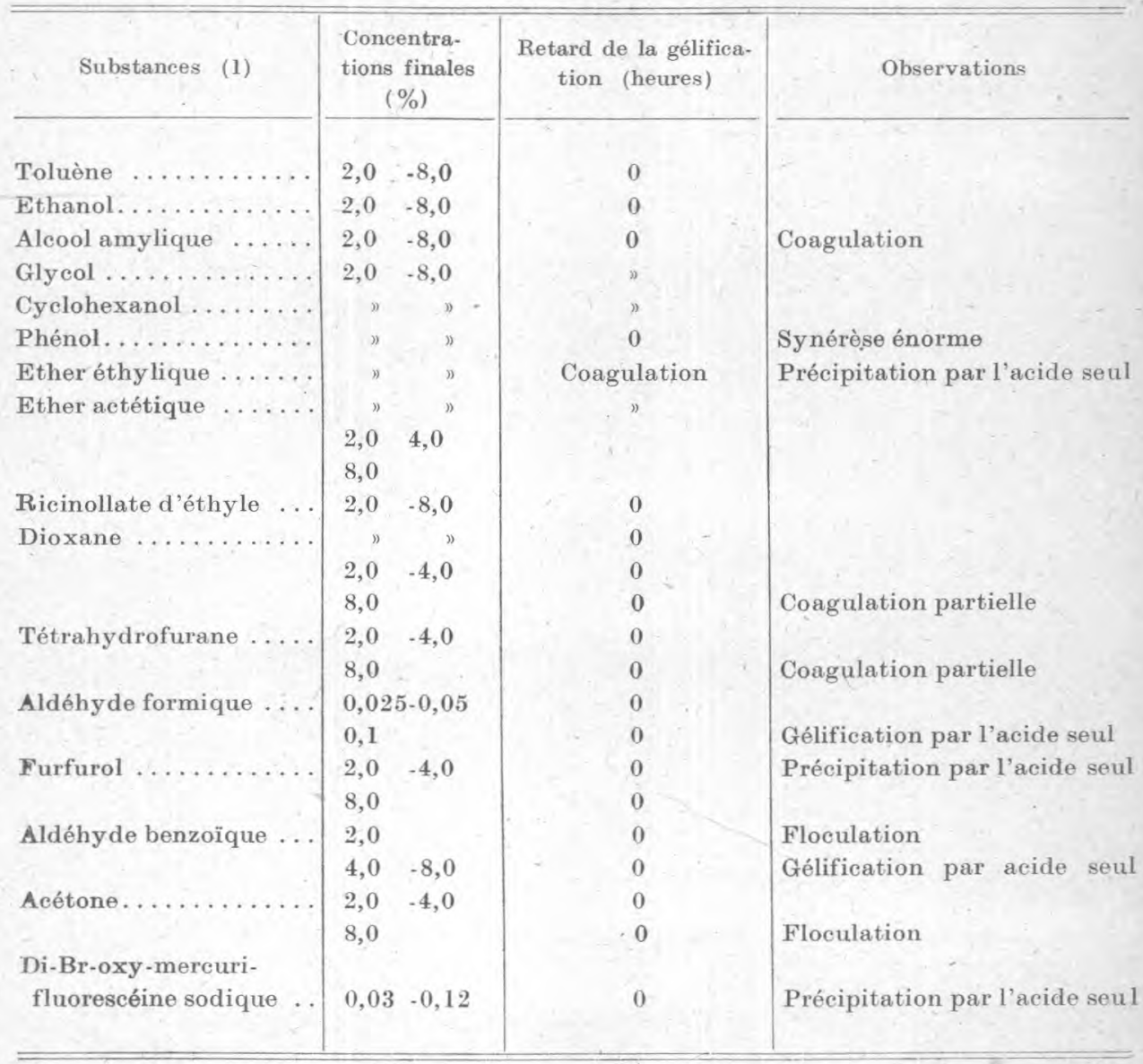

(1) Voir note page précédente.

Comparons ces résultats avec ceux obtenus en étudiant l'action de la présure en présence de mêmes stabilisants.

\section{Stabilisation du lait au contact de la présure}

Les travaux concernant cette question sont nombreux, mais très contradictoires. Signalons quelques résultats concordants. D'après GERBER, en 1907, les fluorures, les oxalates et les phosphates arrêtent l'action de la présure ; d'après KaUfFManN, le thymol et le toluène n'exercent pour ainsi dire aucune action, tandis que le tanin arrête nettement la catalyse en question ; pour SchomndoRfF et Victorow, l'effet produit par l'éthanol, l'éther éthylique et l'acétone serait insignifiant ; PfLeiderer avance que 
l'action de l'acide chlorhydrique est favorisante, mais que celle des acides borique, lactique, acétique et sulfurique serait nulle; mais ces résultats ne sont pas d'accord avec les recherches anciennes de MAYER ; enfin, d'après HATA le bichlorure de mercure arrête l'action de la présure mais ne détruit pas ce ferment.

Nous avons repris cette étude, en l'élargissant sur toutes les substances précédemment utilisées en tant que stabilisant le lait envers l'activité des microorganismes et envers l'acide lactique. Nous avons travaillé dans les mêmes conditions, c'est-à-dire avec du lait frais (mélange livré aux laiteries parisiennes et utilisé dans la journée de la livraison) ; la présure liquide provenait des Usines Fabre à Paris : on en prenait une goutte pour $5 \mathrm{~cm}^{3}$ de lait ; la température ambiante variait dans les limites de 17-240 $\mathrm{C}$.

Voici les résultats résumés sous forme de tableau ci-dessous :

TABLEAU XLVII

STABILISATION DU LAIT SOUMIS A L'ACTION DE LA PRÉSURE

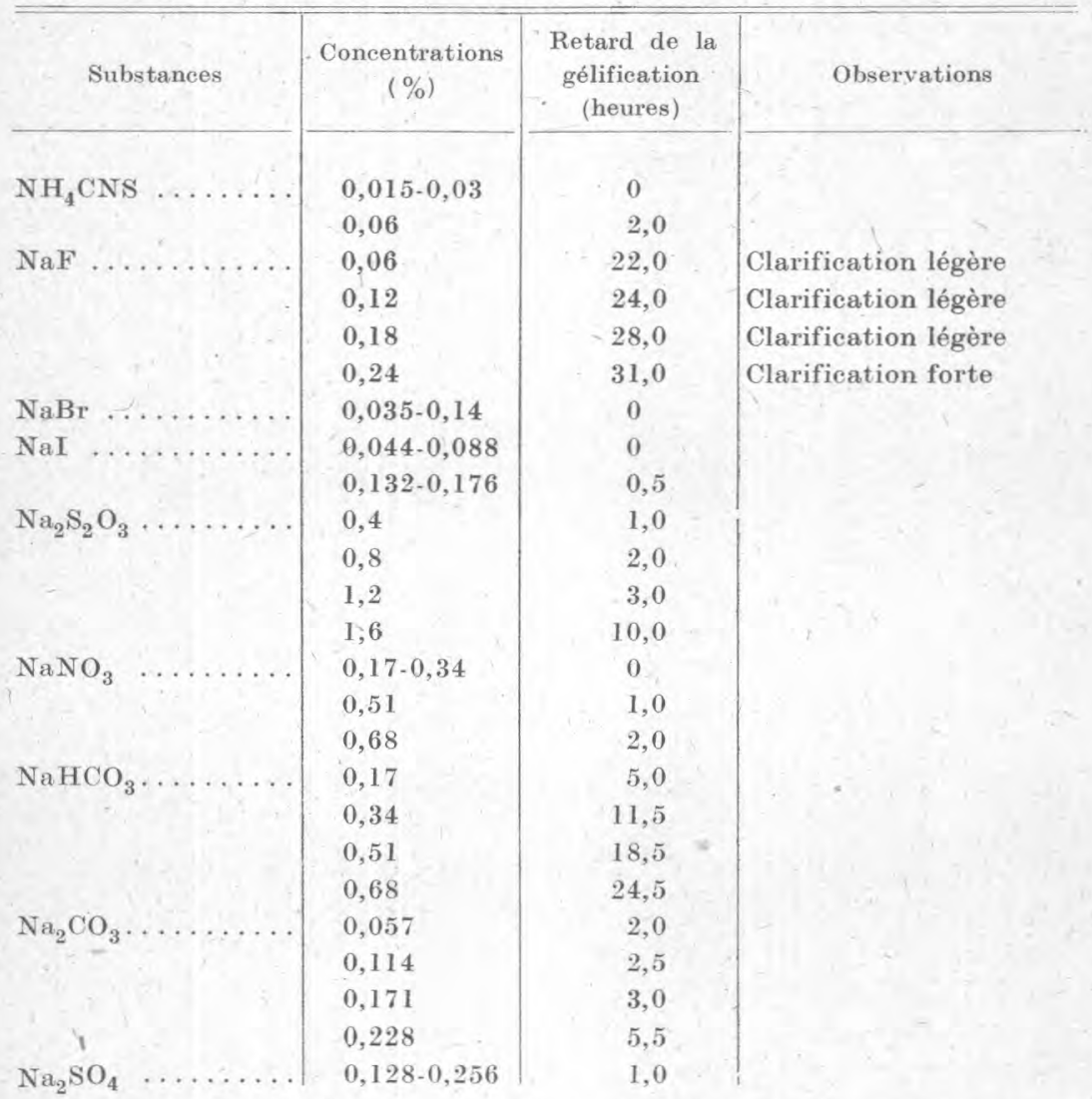


TABLEAU (suite)

\begin{tabular}{|c|c|c|c|}
\hline Substances & $\begin{array}{c}\text { Concentrations } \\
(\%)\end{array}$ & $\begin{array}{l}\text { Retard de la } \\
\text { gélification } \\
\text { (heures) }\end{array}$ & Observations \\
\hline $\mathrm{Na}_{2} \mathrm{HPO}_{4} \ldots$ & $0,072-0,288$ & 1,5 & \\
\hline $\mathrm{Na}_{3} \mathrm{PO}_{4} \ldots \ldots \ldots$ & $0,08-0,16$ & 0 & \\
\hline & $0,24-0,32$ & 2,0 & \\
\hline $\mathrm{Na}_{3} \mathrm{FeCN}_{6} \ldots \ldots$ & 0,12 & 5,5 & \\
\hline & 0,24 & 6,5 & \\
\hline & 0,36 & 7,5 & \\
\hline & 0,48 & 9,5 & \\
\hline $\mathrm{Na}_{4} \mathrm{FeCN}_{6} \ldots \ldots \ldots$ & 0,10 & 2,5 & \\
\hline & 0,2 & 3,5 & \\
\hline & 0,3 & 4,5 & \\
\hline & 0,4 & 5,5 & \\
\hline $\mathrm{AgNO}{ }_{3} \ldots \ldots \ldots$ & $0,02-0,08$ & 0 & Coagulation \\
\hline $\mathrm{CaCl}_{2}{ }^{\circ} \ldots \ldots \ldots \ldots$ & $0,44-1,76$ & 0 & Gel en quelques minutes \\
\hline $\mathrm{HgCl}_{2} \ldots \ldots \ldots \ldots$ & 0,1 & 96,0 & \\
\hline $\mathrm{CuCl}_{2} \ldots \ldots \ldots \ldots$ & $0,07-0,28$ & 0 & Gel en quelques minutes \\
\hline $\mathrm{PbCl}_{2} \ldots \ldots \ldots \ldots$ & $0,012-0,048$ & 0 & Gel en quelques minutes \\
\hline Pb-acétate ...... & $0,03-0,12$ & 0 & Gel en quelques minutes \\
\hline Sels biliaires . . . . . . & 0,1 & 1,0 & \\
\hline & 0,2 & 1,5 & \\
\hline & 0,3 & 2,5 & \\
\hline & 0,4 & 3,0 & \\
\hline Chloramine $\mathbf{T}$ & 0,2 & 38,0 & \\
\hline & 0,4 & 80,0 & \\
\hline Hexaméthy lène- & & & \\
\hline tétramine .... & $0,1-0,4$ & 1,5 & \\
\hline Aniline $\ldots . . .$. & 2,0 & 0 & \\
\hline & 4,0 & 0,5 & \\
\hline & 6,0 & 72,0 & \\
\hline & 8,0 & 216,0 & Coagulation \\
\hline Pyridine $\ldots$. & 2,0 & 0 & \\
\hline & 4,0 & 6,0 & \\
\hline & 6,0 & au delà de & \\
\hline & & 15 jours & \\
\hline Nicotine . & 0,5 & $\begin{array}{l}\text { au delà de } \\
6 \text { jours }\end{array}$ & $\begin{array}{l}\text { Décoloration avee } \\
\text { floculation }\end{array}$ \\
\hline $\begin{array}{l}\text { Ether de pétrole ... } \\
\left(40-55^{\circ} \mathrm{C} .\right)\end{array}$ & $2,0=8,0$ & 0 & \\
\hline Benzène $\ldots \ldots \ldots$. & $2,0-8,0$ & 0 & \\
\hline Toluène .......... & $2,0-8,0$ & 0 & \\
\hline Ethanol ...... & $2,0-8,0$ & 0 & \\
\hline Alcool amylique ... & $2,0-8,0$ & 0 & Gels instantanés \\
\hline Glycol............ & $2,0-8,0$ & 0 & Gels en quelq. minute \\
\hline
\end{tabular}


TABLEAU ( $f$ in $)$

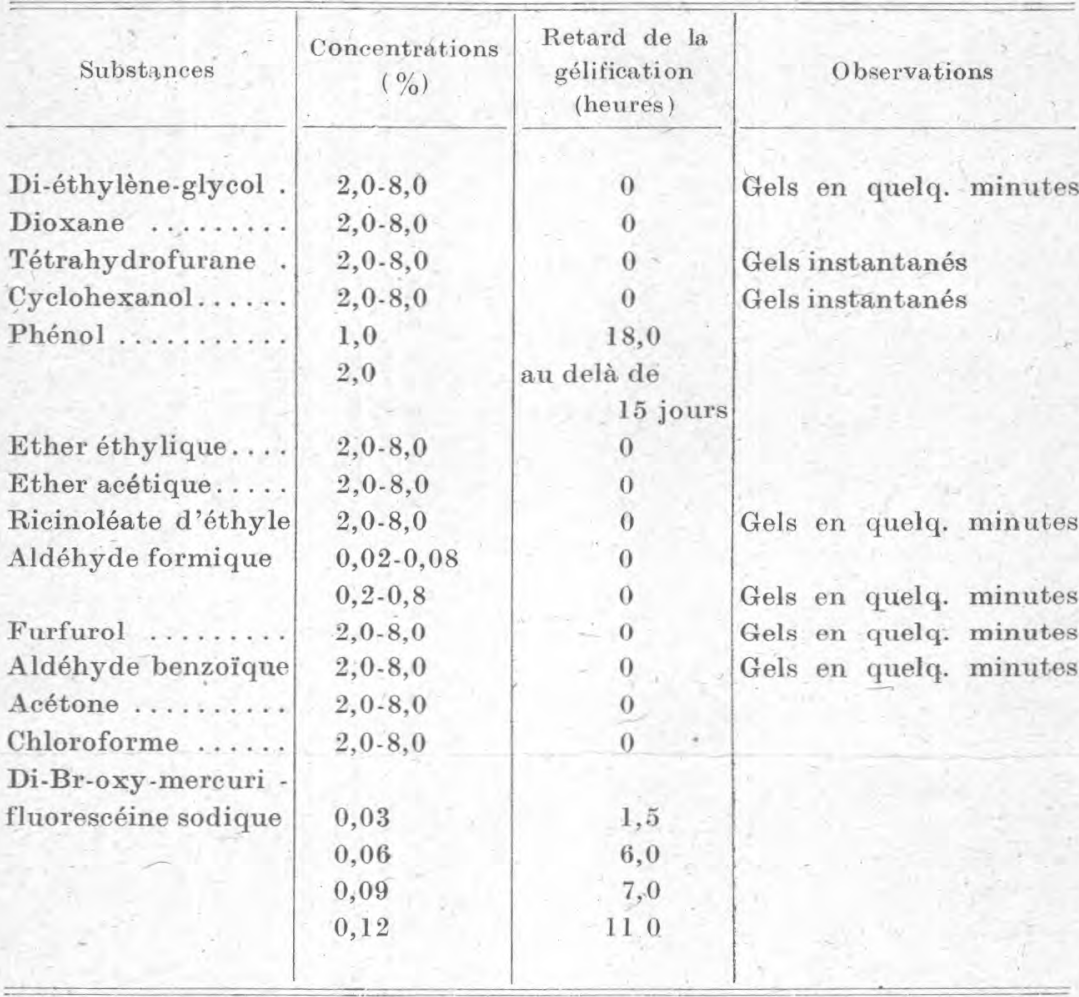

Ayant ainsi étudié la protection du lait à l'égard des agents gélifiants tels que les microorganismes, l'acide lactique pur et la présure, nous allons récapituler ces résultats afin de rechercher les substances ayant un effet stabilisant dans les trois cas étudiés (tableau XLVIII).

TABLEAU XLVIII

STABILISATION DU LAIT GONTRE L'AGTION GELLIFIANTE

\begin{tabular}{|c|c|c|c|c|}
\hline Stabilisants & Microbes & $\begin{array}{c}\text { Acide } \\
\text { lactique }\end{array}$ & Présure & Observations \\
\hline Anions : & & & & . \\
\hline $\mathrm{NaCl} \ldots \ldots \ldots \ldots \ldots \ldots$ & + & 0 & + & . \\
\hline $\mathrm{NaF} \ldots \ldots \ldots \ldots \ldots$ & + & 0 & + & Phénomènes secondaires \\
\hline NaI $\ldots \ldots \ldots \ldots \ldots \ldots$ & + & 0 & + & \\
\hline $\mathrm{NaHCO}_{3} \quad \ldots \ldots \ldots \ldots$ & + & + & + & \\
\hline $\begin{array}{l}\mathrm{Na}_{2} \mathrm{CO}_{3} \ldots \ldots \ldots \ldots \ldots \ldots \ldots \\
\mathrm{Na}_{2} \mathrm{HPO}_{4} \ldots \ldots \ldots \ldots \ldots \ldots\end{array}$ & $\begin{array}{l}+ \\
+\end{array}$ & $\begin{array}{r}0 \\
+ \\
+\end{array}$ & + & Phénomènes secondaires \\
\hline
\end{tabular}


TABLEAU (suite)

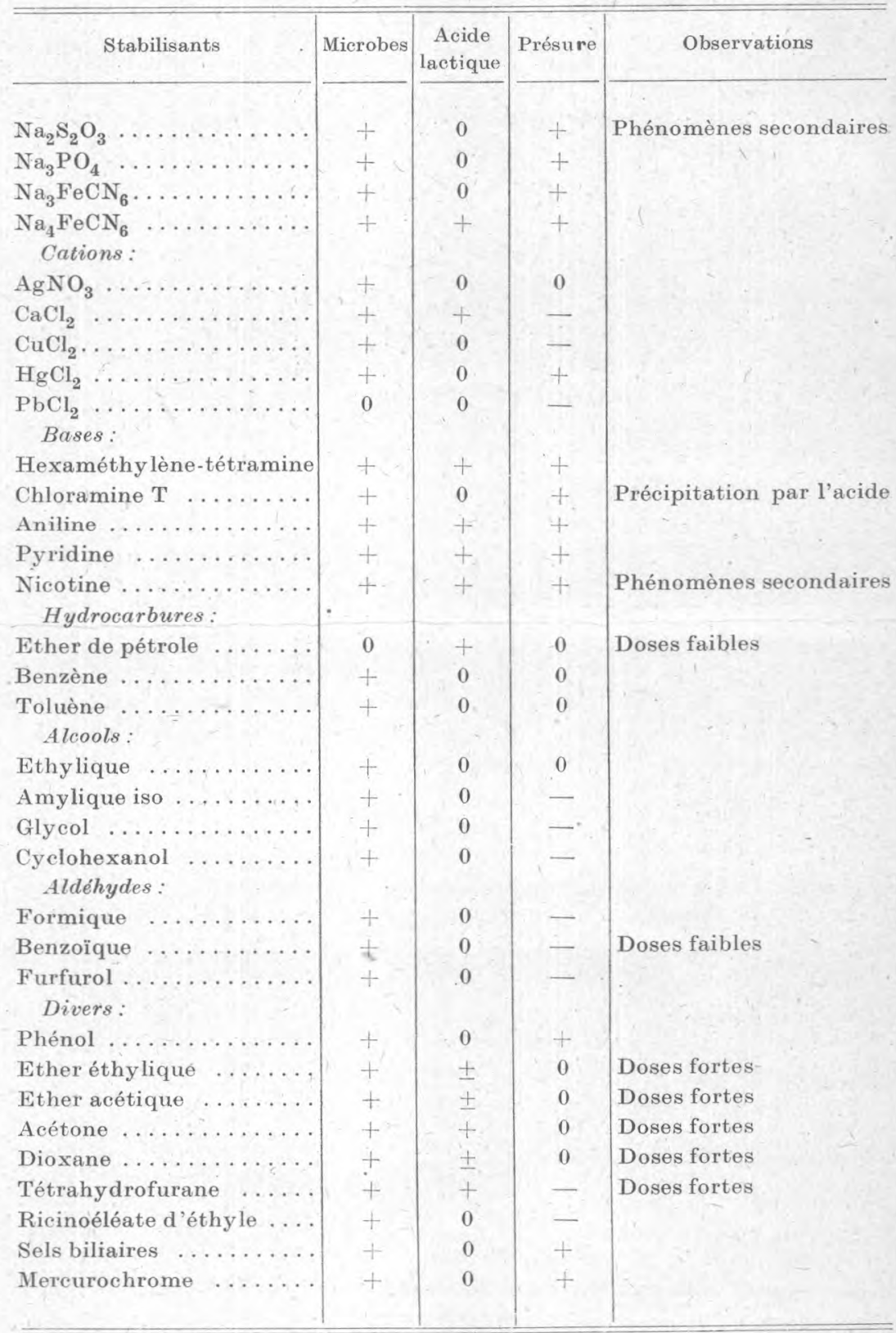
tion.

- - Stabilisation; 0 - sans effet ; - ace élérat ion de la gélification; \pm coagula- 
En présence de ces résultats nous pouvons aborder l'examen du mécanisme de la stabilisation du lait.

\section{Mécanisme de la stabilisation}

Essayons de comprendre le mécanisme de la stabilisation du lait à la lumière des faits que nous venons de découvrir.

Trois hypothèses viennent à l'esprit : biologique, chimique ou physique.

Le mode de voir purement biologique prédomine actuellement : on pense, avant tout, au pouvoir antiseptique des substances employées, et c'est en utilisant les antiseptiques que l'on pense pouvoir résoudre le problème de la stabilisation du lait soumis à l'action gélifiante des produits du métabolisme microbien. Nous allons démontrer que l'effet antiseptique ne présente qu'un aspect du problème.

Dressons, tout d'abord, une liste des antiseptiques variés, tout en soulignant que les données s'écartent parfois sensiblement d'un auteur à l'autre, sans parler d'une bactérie à l'autre. Or, ce qui nous intéresse à ce point de vue ce sont les microbes dont les produits de métabolisme gélifient le lait. On y distingue deux groupes de microorganismes: ceux qui se trouvent normalement dans le canal central des trayons et ceux qui polluent le lait une fois sorti des trayons. Au premier groupe appartiennent divers microbes lactiques (microcoques et bacilles); ils ont été décrits, bien avant Pasteur, en 1847, par Blondeau. Le deuxième groupe est formé par des microbes venus du dehors et ils sont d'autant plus nombreux et d'autant plus variés que les conditions hygiéniques qui président à la traite sont moins correctes; parmi ces microbes citons B. mégatherium, mycoides, sphericus, pyocyaneus, prodigiosus, et, malheureusement trop fréquemment, Bac. Coli.

Il faut souligner que le pouvoir antiseptique des diverses substances à leur égard n'a pas été étudié à fond. Contentons-nous donc de récapituler quelques données signalées par MrQUEL, BEHRInc, Flemming, Zunz et autres (tableau XLIX ci-après).

Rappelons que CuSO et $\mathrm{HgCl}^{2}$ arrêtent la prolifération des staphylocoques en concentration $10^{-7}, \mathrm{AgNo}^{3}$ en $10^{-6}$, chloramine en $10^{-4}$ et $\mathrm{NaF}$ en celle de $10^{-3}$.

Ajoutons à cette liste les essences dont le pouvoir antiseptique a été étudié en détails par Moret et Rochaix en 1925 ; leur effet décroît en partant de thym, citron, genièvre, menthe, goménol, orange, citronelle, girofle, lavande, romarin, bergamotte, eucalyptus, santal, anis et badiane. 
TABLEAU XLIX

POUVOIR ANTISEPTIQUES DES QUELQUES SUBSTANGES CHIMIQUES (GOEFFIGIENT PHÉNOL)

\begin{tabular}{|c|c|c|c|}
\hline Substances & Miquel & Behring & Flemming \\
\hline Eau oxygénée. & 64,0 & - & 2,0 \\
\hline Sublimé........... & 46,0 & 0,016 & $0,1 \quad$ (Zunz) \\
\hline Chloramine .... & 6,0 (Dakin) & - & 3,0 \\
\hline Thymol $\ldots . .$. & 2,5 & 2,0 & - \\
\hline Chloroforme $\ldots \ldots \ldots$. & 4,0 & - & 500,0 \\
\hline Acide salieylique...$\ldots$. & 3,2 & 3,0 & 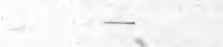 \\
\hline Cyanure de $K \ldots \ldots \ldots$ & 2,7 & - & - \\
\hline Bichromate de K ........ & 2,7 & - & - \\
\hline $\mathrm{ZnCl}_{2} \ldots \ldots \ldots \ldots \ldots$ & 1,7 & - & - \\
\hline Nitrobenzène . . . . . . . & 1,2 & 二 & - \\
\hline Benzène ....... & 1,0 & $\rightarrow$ & - \\
\hline Phénol.............. & 1,0 & 1,0 & 1,0 \\
\hline Permanganate de $\mathbf{K} \ldots$ & 0,9 & - & 1,0 \\
\hline Tanin $\ldots \ldots \ldots \ldots$ & 0,6 & - & $=\div-\div$ \\
\hline Acide borique...$\ldots \ldots$ & 0,4 & - & $2,0 \quad$ (Zunz) \\
\hline Oxyde d'éthyle ........ & 0,14 & - & $.500,0$ \\
\hline Ethanol . . . . . . . . & 0,03 & $=$ & \\
\hline
\end{tabular}

Rappelons enfin que de nombreuses matières colorantes possèdent un effet antibiotique manifeste ; elles ont été étudiées déjà en 1889 par Stilling et depuis par Browning, Zunz, Doladilhe et autres. Voici, d'après Zunz, le pouvoir antibiotique de quelques colorants :

Colorants

Auramine

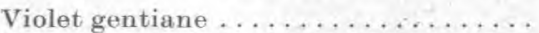

Vert malachite ................

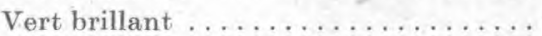

Bleu de méthy lène ..............

Jaune d'acridine
Doses toxiques
$1 / 5.000$
$1 / 5.000$
$1 / 2.000$
$1 / 1.000$
$1 / 1.000$
$1 / 500$

Les chiffres donnés par BrownING s'écartent des précédents. D'après certaines indications, le rouge-neutre, le rouge de Congo et le bleu d'indigo n'auraient aucun pouvoir antiseptique. Lorsque nous comparons ces résultats avec ceux que nous avons signalés dans le chapitre précédent notamment en parlant de l'action retardataire sur la gélification du lait par les microbes, nous constatons que les matières en question s'y retrouvent ; peut être, le retard apporté par ces colorants à la gélification du lait serait dû donc, en partie du moins, à leur effet antiseptique. Mais, nous devons remarquer aussi, que les colorants en question sont basiques, à l'exception 
du vert malachite et de l'auramine et que par conséquent les phénomènes de fixation peuvent intervenir (1).

Nous allons compléter cette étude en utilisant les antibiotiques récents tels que la pénicilline, les sulfamides et les essences, etc.

Les résultats de nos recherches sont résumés dans le tableau ci-dessous.

TABLEAU L

LES ANTISEPTIQUES ET LA STABILISATION DU LAIT

\begin{tabular}{|c|c|c|}
\hline Substances & $\begin{array}{c}\text { Concentrations } \\
(\%)\end{array}$ & $\begin{array}{c}\text { Retard de la gélification } \\
\text { (jours) }\end{array}$ \\
\hline \multirow[t]{4}{*}{ Essence de thym } & XX gouttes & 0,3 \\
\hline & $\mathrm{XL}$ & 2,5 \\
\hline & $\mathrm{Lx}$ & 3,8 \\
\hline & LXXX & 4,2 \\
\hline \multirow[t]{4}{*}{ Essence de menthe...$\ldots$. } & $\mathrm{xx}$ & 0,2 \\
\hline & $\mathrm{XL}$ & 0,4 \\
\hline & LX & 2,0 . \\
\hline & LXXX & 2,0 \\
\hline \multirow[t]{4}{*}{ Essence de niaouli. . } & $\mathrm{xx}$ & 0,5 \\
\hline & $X L$ & 0,7 \\
\hline & LX & 0,9 \\
\hline & LXXX & 2,5 \\
\hline \multirow[t]{4}{*}{ Essence d'aspic } & $\mathrm{xx}$ & 0,2 \\
\hline & $\mathrm{XL}$ & 0,3 \\
\hline & LX & 0,6 \\
\hline & LXXX & 2,0 \\
\hline \multirow[t]{2}{*}{ Menthol (sol. aq. sat. $15^{\circ}$ C.) . } & 1,0 à $2,0 \mathrm{~cm}^{3}$ & 0 \\
\hline & $3,0-4,0 \mathrm{~cm}^{3}$ & 0,3 \\
\hline Thymol (sol. aq. sat. à $15^{\circ} \mathrm{C}$.) & $1,0-4,0 \mathrm{~cm}^{3}$ & 0,2 \\
\hline \multirow[t]{4}{*}{ Pénieilline $\mathrm{G} \ldots \ldots \ldots \ldots \ldots$} & 0,06 & 1,7 \\
\hline & 0,11 & 1,7 \\
\hline & 0,16 & 1,0 \\
\hline & 0,22 & 1,5 \\
\hline \multirow[t]{4}{*}{ p-amino-phényl-sulfamide .. } & 0,02 & 0,1 \\
\hline & 0,04 & 0,5 \\
\hline & 0,08 & 1,0 \\
\hline & 0,10 & 2,0 \\
\hline \multicolumn{3}{|l|}{ Sulfadiazine } \\
\hline (sol. aq. sat. à $15^{\circ}$ C.) $\ldots$ & $2,0-10,0 \mathrm{~cm}^{3}$ & 0 \\
\hline \multirow[t]{4}{*}{ Sulfaguanidine . . } & 0,002 & 1,0 \\
\hline & 0,004 & 1,2 \\
\hline & 0,008 & 1,5 \\
\hline & 0,010 & 1,6 \\
\hline Iode (sol. aq. sat. à $15^{\circ}$ C.) ... & 0,2 à 5 mierogrammes & 0,2 \\
\hline
\end{tabular}


Il est intéressant de classer toutes les substances antibiotiques selon leur action sur la gélification microbienne du lait :

TABLEAU LI

AGTIVITÉ DES SUBSTANCES ANTIBIOTIQUES

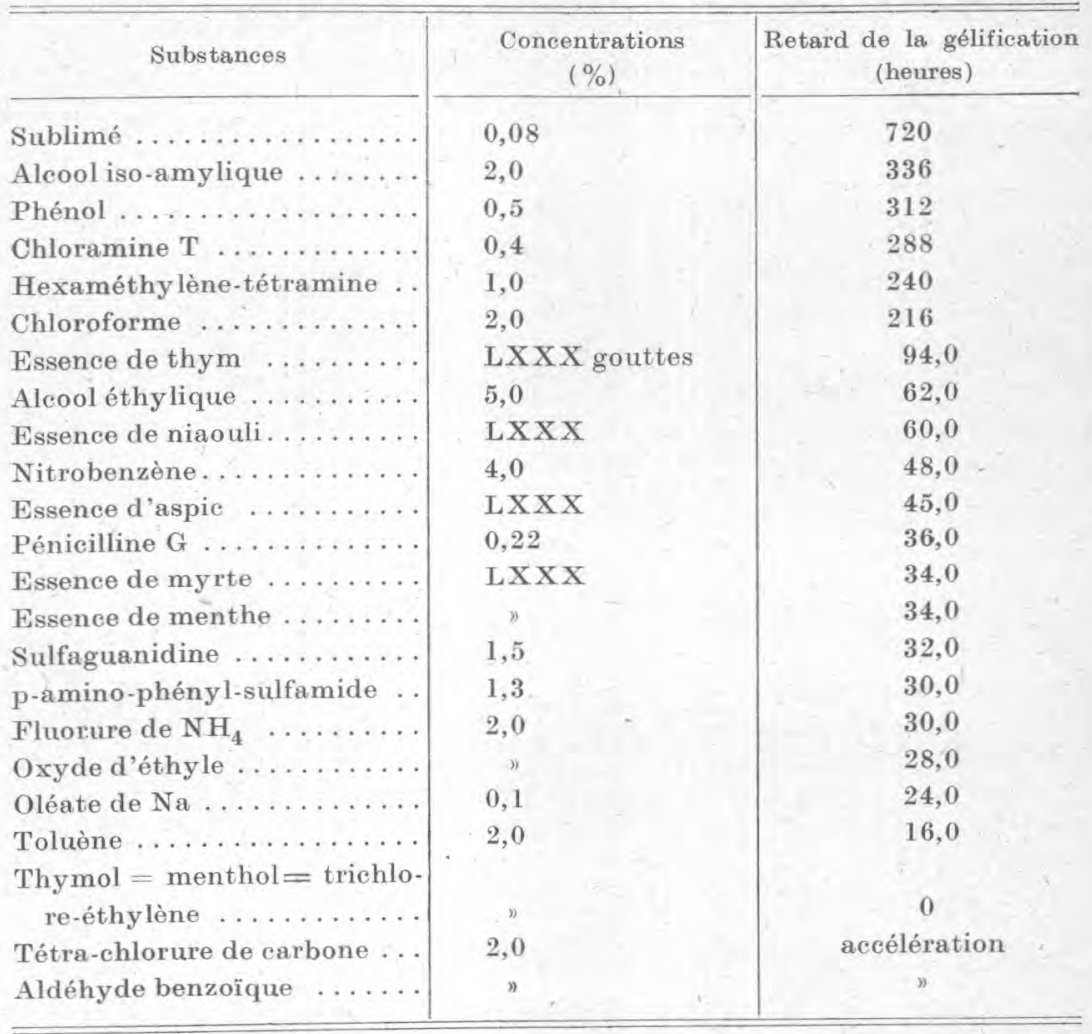

Notes de la page 491.

(1) Doladilhe et Guy ont pensé que le pouvoir antiseptique des colorants serait dû à leur adsorption par les microbes tels que le staphylocoque blane, streptocoques, entérocoques, Coli, pyocyanique, diphtérique, typhique, paratyphique, ete. ; ils considèrent ces microbes comme portant une charge électrique négative; or, nous avons montré en 1931 que cette charge électrique dépend de leur âge et de la nature du milieu nutritif. Les auteurs en question n'ont pas vérifié la nature électrique de leurs colorants : en effet, on ne peut parler de charge électrique que pour les colloïdes typiques ; or, à ce point de vue leurs matières colorantes sont, en grande partie dialysables (jaune d'acridine, auramine, violet de gentiane, trypaflavine, etc.) ; en outre, le vert malachite est un colorant acide et non basique. Les conelusions des auteurs sont done à reprendre.

(2) Il convient de noter que le thymol, en solution aqueuse saturée à $15^{\circ} \mathrm{C}$., augmente l'action retardatrice des diverses substances antibiotiques étudiées : il double celle de chloramine à $2,0 \%$, du sublimé à $0,0033 \%$, d'iodure et de bromure de sodium ainsi que d'hyposulfite de sodium à $0,84 \%$. 
Pour comprendre l'action des antibiotiques étudiés nous allons relater quelques faits qui nous paraissent intéressants.

Tout d'abord, l'action de certaines substances étudiées n'empêchent pas le développement dés divers champignons au bout d'un certain temps de contact : ainsi après 6 jours on constate leur apparition en présence de l'eau oxygénée (en concentration finale de $4,0 \%$ ), au bout de 7 jours en présence de citrate ou d'iodate de $\mathrm{Na}$ en concentration respectives de 0,96 et de $0,16 \%$ et au bout de 10 jours au contact de sulfite de $\mathrm{Na}(0,32 \%)$, de l'hyposulfite $(1,76 \%)$, de fluorure de $\mathrm{NH}^{4}(0,44 \%)$ et de sulfocyanure de $\mathrm{NH}^{4}$ $(1,14 \%)$.

L'action de la pénicilline et des sulfamides étudiés permet de constater que ces antibiotiques agissent électivement sur certains microorganismes. Le lait conservé liquide, grâce à la présence de la pénicilline $\mathrm{G}$ durant 48 heures contient des colibacilles (coagulation. du lait, pas de liquéfaction de la gélatine, production d'indol, caractères morphologiques et tinctoriaux), ainsi que des bacilles ayant quelques caractères de paratyphiques (pas de liquéfaction de la gélatine, pas de coagulation du lait, pas de production d'indol, caractères morphologiques et tinctoriaux). On a pu isoler du lait, resté liquide en présence de p-amino-phényl-sulfamide, d'une part, un bacille rappelant celui de Friedlaender, mais légèrement mobile et sans capsules même cultivé sur la gélatine, ne liquéfiant pas cette dernière, coagulant le lait et ne produisant que des traces d'indol en 48 heures et, d'autre part, un bàcille type " proteus vulgaris" mais à colonies limitées peu extensives, liquéfiant la gélatine, coagulant le lait avec production d'acide et d'indol (1).

Enfin, fait intéressant, la pénicilline se montre d'une activité périodique en fonction des concentrations :

$\begin{array}{cc}\begin{array}{c}\text { Concentrations } \\ (\%)\end{array} & \begin{array}{c}\text { Retard de la gélification } \\ \text { (heures) }\end{array} \\ 0,0057 & 36,0 \\ 0,110 & 36,0 \\ 0,136 & 18,0 \\ 0,161 & 24,0 \\ 0,211 & 30,0 \\ 0,230 & 40,0\end{array}$

Cette dernière conclusion mérite, toutefois, d'être approfondie, étant donné les variations de la gélification selon la provenance du lait, la température, le diamètre de tubes à essais employés, etc.

De ces résultats il est permis de tirer la conclusion que les antibiotiques les plus énergiques, tels que la pénicilline ou les sulfamides

(1) Nous remercions vivement $M$. Beauvy de sa collaboration empressée. 
n'ont qu'un effet relativement faible lorsqu'on le compare avec celui que l'on observe avec d'autres substances dont le pouvoir antiseptique est nul et que nous allons relater dans les pages suivantes.

Il s'ensuit donc que le pouvoir antibiotique ne règle pas, à lui tout seul, la stabilité du lait.

En effet, le lait se gélifie plus facilement pendant les orages, par la chauffe à $130^{\circ} \mathrm{C}$. dans des tubes scellés, et par un biocatalyseur, la présure, sans aucune acidification concomitante.

Ce dernier fait nous amène à souligner quelques points essentiels concernant la catalyse. Rappelons tout d'abord que le catalyseur accélère une réaction qui se poursuit normalement d'elle-même mais très lentement; que l'activité d'un catalyseur dépend du degré de dispersion de ce dernier, ainsi que l'ont démontré RuszNYAK pour les métaux colloïdaux et FODOR pour la levure ; que l'effet catalytique est tributaire de la réaction réelle du milieu, de la température, des radiations, des phénomènes électriques, etc., et que chacun de ces facteurs se trouve souvent sous la dépendance des autres, ce qui rend cette étude expérimentale difficile et les conclusions problématiques, ainsi que nous l'avons souligné à plusieurs reprises (Traité de Biocolloüdologie, t. III, p. 1-81). De plus, l'arrêt d'un processus biocatalytique - gélification du lait par la présure - peut être amené par des "poisons catalytiques ". Parmi ces "poisons" citons l'ozone, les rayons ultra-violets, l'aldéhyde formique, l'éthanol, le thymol (FrEUDENREICH, en 1898), le chlorure de vanadium (RICHET, en 1908), etc. Voici, d'après BrEDIG, les concentrations finales qui empêchent l'activité de la catalase du sang :

TABLEAU LII

" POISONS" GATAlytoues (GRAMME MOLÉGule PAR litre)

\begin{tabular}{|c|c|}
\hline Substances & Concentrations finales \\
\hline $\mathrm{HCN} \ldots \ldots \ldots \ldots \ldots \ldots \ldots$ & $5,0-10^{8}$ \\
\hline 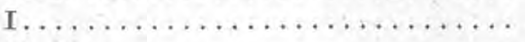 & $1,0-10-7$ \\
\hline$\ldots \ldots \ldots \ldots \ldots \ldots \ldots \ldots$ & $1,0 \cdot 10.6$ \\
\hline $\mathrm{Br}=\mathrm{NH}_{4}=\mathrm{P} \ldots \ldots \ldots \ldots \ldots$ & $4,0-10.5$ \\
\hline $\mathrm{C}_{2} \mathrm{H}_{5}-\mathrm{NH}_{2} \ldots \ldots \ldots \ldots \ldots \ldots$ & $18,0 \cdot 10-5$ \\
\hline$\ldots \ldots \ldots \ldots \ldots \ldots \ldots \ldots$ & $20,0=10-^{5}$. \\
\hline$\ldots \ldots \ldots \ldots \ldots \ldots$ & $24,0-10-^{5}$ \\
\hline $\mathrm{Na}_{2} \mathrm{~S}_{2} \mathrm{O}_{3} \ldots \ldots \ldots \ldots \ldots \ldots \ldots$ & $2,0-10-4$ \\
\hline $\mathrm{HCl} \ldots \ldots \ldots \ldots \ldots \ldots \ldots \ldots$ & $3,0-10-4$ \\
\hline $\mathrm{NaS}_{2} \mathrm{O}_{3} \ldots \ldots \ldots \ldots \ldots \ldots$ & $2,0 \cdot 10.3$ \\
\hline $\mathrm{C}_{2} \mathrm{H}_{2} \mathrm{O}_{4} \ldots \ldots \ldots \ldots \ldots \ldots$ & $1,0-10 \cdot 3$ \\
\hline $\mathrm{HgCN}_{2} \ldots \ldots \ldots \ldots \ldots \ldots \ldots \ldots$ & $48,0 \cdot 10 \cdot 2$ \\
\hline
\end{tabular}

Notons que ces substances arrêtent la catalyse par leur seule présence, mais ne détruisent pas le catalyseur : une fois éliminée, la catalyse reprend. De plus, leur action est périodique, RıcHet 
l'a démontré sur la fermentation lactique en présence du chlorure de vanadium : en concentration finale de $10-^{-4}$ à $10^{-5}$ ce sel ralentit la catalyse en question ; en concentration $10^{-5}$ à $10_{-6}^{6}$ il y a une accélération; à $10-^{7}-$ un ralentissement et à $10-^{8}$ - une nouvelle accélération.

En résumé, nous retrouvons dans la liste des "poisons" catalytiques connus plusieurs substances ayant en même temps un pouvoir antibiotique.

Mais les termes "antiseptiques", "poisons", "catalytiques", " antibiotiques ", ne cachent que notre ignorance du mécanisme du phénomène. En effet, quel est ce mécanisme? Assurément polyvalent. On a mis en avant des phénomènes variés : osmotique, deshydratations cellulaires $\left(\mathrm{KCl}, \mathrm{NaCl}, \mathrm{KNO}^{3}\right.$ en solutions concentrées), oxydation ( $\mathrm{KMnO}^{4}, \mathrm{Cl}, \mathrm{I}$, etc.), mais aussi réduction (aldéhyde formique, $\mathrm{SO}^{2}$, etc.), car l'hydrogène in statu nascendi s'oppose à la multiplication cellulaire ; on a accusé les réactions de floculations des protides (sels des métaux lourds), et adsorption élective, certains antiseptiques se scindent dans l'organisme en donnant des produits actifs (hexaméthylène-tatramine). Mais on n'a envisagé ni les processus de la lyse microbienne, ni les troubles de la perméabilité des cellules vivantes. En somme, le problème n'a pas encore été suffisamment creusé et le pouvoir antiseptique reste inexpliqué, tout comme celui des "anticatalyseurs".

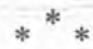

La stabilisation du lait peut être comprise dans certains cas par des mécanismes purement chimiques ou physicochimiquesl

En ce qui concerne l'activité microbienne dans sa répercussion sur l'équilibre du lait et notamment sa gélification, plusieurs phénomènes peuvent y être démêlés. Tout d'abord l'action gélifiante des acides issus de l'activité microbienne. On peut donc penser qu'en renforçant le pouvoir régulateur du lait on pourrait empêcher cette gélification et, eo ipso, stabiliser ce, complexe biologique. Nous avons ajouté un tampon approprié afin de maintenir le lait au $p \mathbf{H}$ $=6,3$; c'est le tampon de Mc. Ilvaine composé, comme on sait, de phosphate bisodique et d'acide nitrique ; voici les résultats obtenus :

STABILISATION DU LAIT PAR LE TAMPON DE MC. ILVAINE

$\begin{array}{cc}\begin{array}{c}\text { Coneentrations finales } \\ (\%)\end{array} & \begin{array}{c}\text { Retard de la gélifieation } \\ \text { (jours) }\end{array} \\ 2,5 & 0 \\ 5,0 & 0,2 \\ 10,0 & 0,3 \\ 20,0 & 1,5\end{array}$


Done, en renforçant ce pouvoir régulateur du lait on retarde un peu la gélification.

On pouvait également penser que l'addition des bases, donc une neutralisation chimique, empêcherait la gélification du lait de se produire. C'est également le cas : nous avons donné dans des tableaux précédents, un certain nombre de bases qui s'opposent à la gélification du lait (diméthyl et di-éthyl-amines, triéthanol-amine ainsi que les sels alcalins, tels que le bicarbonate et le carbonate de sodium, etc.). D'autres bases possèdent un pouvoir antigélifiant beaucoup plus énergique (aniline, hexaméthylène-tatramine, pyridine, nicotine, etc.). L'importance de cette neutralisation purement chimique apparaît en étudiantla stabilisation du lait soumis à l'action de l'acide lactique : ce sont, avant tout, les bases et les sels alcalins qui suppriment nettement cette gélification et même toute coagulation; les autres substances, telles que l'éther de pétrole, le tétrahydrofurane ou l'acétone permettent uniquement d'empêcher la prise en masse, mais il se fait une coagulation plus ou moins complète et plus ou moins fluide. Par conséquent, dans la gélification acide du lait, quelle que soit l'origine de cet acide, le phénomène de neutralisation chimique entre en jeu, mais n'explique pas tout. En effet, l'addition concomitante de la soude caustique, base forte, non seulement n'empêche pas la gélification, mais, bien au contraire, elle la provoque plus rapidement encore; l'action de deux réactifs s'additionne, tout comme dans les cas de la gélification des dispersions sodiques de caséine par l'acide lactique, ainsi que nous l'avons constaté en 1936.

Enfin, en connexion avec l'activité microbienne, nous avons mentionné, le pouvoir réducteur. On pourrait done admettre l'action stabilisante de substances oxydantes. Parmi ces substances citons l'hypochlorite de sodium, le permanganate de potassium, l'eau oxygénée, l'eau de chlore, l'eau iodée, les nitrates en milieu acide, le chromate et le bichromate de potassium. Or, leur rôle stabilisant a été vu par divers expérimentateurs et, quant ànous, nousl'avons constaté en ce qui concerne le permanganate et l'eau iodée ; mais, ces substances sont en même temps des antiseptiques connus. L'oxydation et l'antisepsie vont, dans certains eas, de pair. Malheureusement, dans d'autres cas, ce sont les réducteurs qui stabilisent le lait : l'hyposulfite et le bisulfite de sodium. Ainsi l'entrave apportée à la production des microorganismes, la neutralisation des acìdes qu'ils engendrent ou du pouvoir réducteur qu'ils font apparaître n'explique pas le phénomène de la stabilisation du lait. 
Le phénomène est donc plus complexe. Examinons les conditions d'équilibre colloïdal du lait. Nous les avons étudiées à maintes reprises (Traité de Biocolloïdologie, t. III, Etat colloïdal et Industrie, t. II) ; on peut ramener les facteurs qui les règlent au nombre de trois ; la charge électrique accentuée et, eo ipso, l'agitation moléculaire forte, la tension interfaciale faible, une viscosité forte. Dans le cas des micelles hydratables il faut tenir compte de mouillabilité laquelle dépend, à son tour de l'orientation moléculaire, autrement dit de la polarité ou de la non polarité moléculaire et, en dernière analyse, du moment électrique des molécules. La pratique industrielle tire profit de ces données, en utilisant les savons ayant une tension interfaciale très faible, ou bien les sulforicinates décomposables par les acides ; en bloquant le groupement carboxylique soit par salification (" humectol »), soit par estérification (" avirol »), BERTsch a obtenu des résultats intéressants ; l'estérification des sulforicinates donne divers produits utilisables ("laurol»), les dérivés de naphtalène aussi ("sapamine »), etc.

L'augmentation de la viscosité ne donne pas de résultats valables industriellement parlant; le maniement de la charge électrique se heurte à des difficultés concernant sa juste appréciation quantitative dans les cas de complexes colloïdaux. L'étude de l'orientation moléculaire est à peine commencée.

Pour démêler le rôle de ces facteurs, énumérons tout d'abord les substances particulièrement énergiques à ce point de vue.

TABLEAU LIII

STABILISANTS ÉNERGIQUES DU LAIT

\begin{tabular}{|c|c|c|}
\hline Substances & Concentrations finales & Retard en jours \\
\hline Cyclohexanol .............. & 0,05 & 7,0 \\
\hline $\mathrm{CuCl}_{2} \ldots \ldots \ldots \ldots \ldots \ldots \ldots$ & 0,08 & 15,0 \\
\hline $\mathrm{HgCl}_{2} \ldots \ldots \ldots \ldots \ldots \ldots \ldots$ & 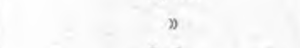 & 30,0 \\
\hline $\mathrm{PdCl}_{2} \ldots \ldots \ldots \ldots \ldots \ldots \ldots$ & 0,1 & 6,0 \\
\hline $\mathrm{NaF} \ldots \ldots \ldots \ldots \ldots \ldots \ldots$ & 0,3 & 3,0 \\
\hline Phénol $\ldots \ldots \ldots \ldots \ldots \ldots \ldots$ & 0,5 & 13,0 \\
\hline NaI $\ldots \ldots \ldots \ldots \ldots \ldots \ldots$ & m & 8,0 \\
\hline Acétate de $\mathrm{Pb} \quad \ldots \ldots \ldots \ldots \ldots$ & 0,6 & 6,0 \\
\hline Chloramine $\mathrm{T} \ldots \ldots \ldots \ldots \ldots \ldots$ & 》 & 12,0 \\
\hline $\mathrm{Na}_{2} \mathrm{~S}_{2} \mathrm{O}_{3} \quad \ldots \ldots \ldots \ldots \ldots \ldots$ & 0,8 & 6,0 \\
\hline $\mathrm{NaSO}_{3} \ldots \ldots \ldots \ldots \ldots \ldots$ & 1,0 & 6,0 \\
\hline $\mathrm{CaCl}_{2} \ldots \ldots \ldots \ldots \ldots \ldots$ & 1,0 & 6,0 \\
\hline 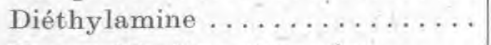 & 1,0 & 4,0 \\
\hline Hexaméthylène-tétramine ...... & 1,0 & 12,0 \\
\hline Aniline $\ldots \ldots \ldots \ldots \ldots \ldots$ & $\quad 1,0$ & 10,0 \\
\hline Pyridine $\ldots \ldots \ldots \ldots \ldots \ldots \ldots$ & n & 12,0 \\
\hline Chloroforme $\ldots \ldots \ldots \ldots \ldots \ldots$ & n & 9,0 \\
\hline Bromoforme $\ldots \ldots \ldots \ldots \ldots \ldots$ & $n$ & 9,0 \\
\hline Tétrahydrofurane $\ldots \ldots \ldots \ldots$. & ) & 9,0 \\
\hline
\end{tabular}


Pour comprendre dans quelle mesure les stabilisants énumérés entrent dans les catégories des stabilisants colloïdaux nous allons donner les caractères électriques et capillaires des substances qui s'opposent à la gélification du lait par l'acidification et par la présure (tableau LIV).

TABLEAU LIV

GARACTÉRES PHYSICO-CHIMIQUES DES STABILISANTS DU LAIT

\begin{tabular}{|c|c|c|c|}
\hline Stabilisants & $\begin{array}{l}\text { Concen- } \\
\text { trations }\end{array}$ & $\begin{array}{c}\text { Tension } \\
\text { superficielle } \\
\text { (dynes/om.) }\end{array}$ & $p \mathrm{H}$ \\
\hline $\mathrm{CaCl}_{2} \ldots \ldots \ldots \ldots \ldots \ldots \ldots \ldots \ldots$ & $\mathrm{M} / 1$ & 72,0 & 4,8 \\
\hline $\mathrm{CuCl}_{2} \quad \ldots \ldots \ldots \ldots \ldots \ldots \ldots$ & $\mathrm{M} / 10$ & 72,6 & 4,8 \\
\hline $\mathrm{HgCl}_{2} \quad \ldots \ldots \ldots \ldots \ldots \ldots \ldots$ & $\mathrm{M} / 10$ & 73,4 & 3,6 \\
\hline 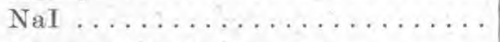 & $\mathbf{M} / \mathbf{1}$ & 71,1 & 11,0 \\
\hline Hyposulfite de Na ............ & $21,0 \%$ & 71,4 & 6,8 \\
\hline Ferrocyanure de sodium $\ldots . . \ldots$ & $\mathrm{M} / 10$ & 72,5 & 8,6 \\
\hline Chloramine $\mathrm{T} \ldots \ldots \ldots \ldots \ldots$ & $10 \%$ & 48,7 & 9,5 \\
\hline Diéthylamine $\quad \ldots \ldots \ldots \ldots \ldots$ & $\mathrm{M} / 10$ & 51,3 & 11,2 \\
\hline Hexaméthy lène tétramine ........ & $10 \%$ & 60,4 & 10,4 \\
\hline Aniline $\ldots \ldots \ldots \ldots \ldots \ldots \ldots$ & pure & 42,5 & 7,8 \\
\hline Pyridine $\ldots \ldots \ldots \ldots \ldots \ldots$ & pure & 38,2 & 8,6 \\
\hline Cyelohexunol $\ldots \ldots \ldots \ldots \ldots \ldots$ & pur & 31,8 & 5,6 \\
\hline Phénol $\ldots \ldots \ldots \ldots \ldots \ldots \ldots$ & hydraté & 38,4 & 4,4 \\
\hline Tétrahydrofurane ............. & * pur & 20,1 & 4,8 \\
\hline Ether de pétrole pur $\ldots \ldots \ldots \ldots$ & pur & 10,5 & 6,0 \\
\hline Phosphate bisodique $\ldots \ldots \ldots \ldots$ & $\mathbf{M} / 10$ & 72,5 & 9,2 \\
\hline
\end{tabular}

On peut immédiatement tirer la conclusion que, les électrolytes mis à part, toutes les autres substances abaissent fortement la tension superficielle. Mais il n'existe pas de parallélisme étroit entre le degré de cet abaissement et l'effet stabilisateur ; toutefois, on ne doit pas conclure trop hâtivement, car la tension superficielle, e'està-dire celle qui existe entre la phase liquide et l'air, ne correspond pas, comme on sait, à celles entre autres phases liquides. Ainsi, la tension entre la phase liquide et l'air pour l'albumine d'œuf est de 69,1 dynes/cm., donc à peine abaissée, tandis que contre l'huile elle tombe à 35,1 dynes $/ \mathrm{cm}$. ; pour l'éthanol, ou pour l'acétone, cet abaissement contre l'huile elle est d'environ la moitié. Néanmoins, malgré ces rectifications éventuelles, on observe des exceptions : ainsi le ricinoléate d'éthyle, l'oléate de sodium, les sels biliaires, l'éther éthylique, etc., substances fortement actives au point de vue capillaire, n'ont qu'un effet stabilisateur faible. De plus, le rôle stabilisateur du chlorure de $\mathrm{Ca}$ ou de ferrocyanure de Na reste 
inexpliqué. L'abaissement de la tension superficielle interfaciale n'est pas le seul et unique facteur intervenant dans le processus de stabilisation.

L'action stabilisante des électrolytes, parfois aussi énergique que celle des diverses autres substances énumérées, fait penser à l'intervention de la charge électrique. On ne doit pas se préoccuper du fait que l'on y rencontre aussi bien les cations que les anions : l'étude du $p \mathrm{H}$ du lait additionné de ces substances semble donner la clef de cette constatation. Les solutions en question sont tantôt acides tantôt alcalines - inférieures à $p \mathrm{H} 5,0$ ou supérieures à $p \mathbf{H}=8,0$. De plus, les gels formés au bout d'un certain temps de contact avec ces stabilisants sont tantôt acides, tantôt alcalins : alcalins en présence de la pyridine, de la soude caustique, des carbonates, etc., faiblement acides ou acides dans d'autres cas. Du reste, nous avons vu que la gélification du lait peut s'obtenir aussi bien par les acides que par les bases : c'est un phénomène périodique. On comprend donc que les stabilisants acides ou alcalins, pourvu qu'ils renforcent la charge électrique des micelles colloïdales du lait en concentrations appropriées, charge primitive ou ren. versée, peuvent avoir un effet stabilisant.

Il nous reste à examiner le rôle d'hydratation micellaire qui a priori doit jouer un rôle primordial dans la stabilisation : empêcher 1 'hydratation des micelles, donc, in fine, la gélification, e'est le but de l'opération envisagée. On peut y parvenir en introduisant des électrolytés facilement hydratables pour leur compte personnel, Parmi ces ions ceux de $\mathrm{Li}$ et de $\mathrm{Na}$ sont au premier rang suivis de loin par $\mathrm{Ca}$ et $\mathrm{Mg}$ et autres. Or, ce sont les ions $\mathrm{Ca}, \mathrm{Cu}, \mathrm{Hg}, \mathrm{Pd}$, peu hydratables qui se placent au premier rang des stabilisants.

Tournons-nous vers le mécanisme de stabilité du complexe colloïdal lactique basé sur l'existence de la couche protectrice autour de chaque micelle. De quelle nature est-elle cette couche? On ne le sait pas exactement. Dans le cas ou elle serait formée par des matières grasses Ia dissolution de ces matières par les solvants de graisses amènerait sa disparition et, eo ipso, la labilisation du complexe-floculation et, éventuellement, la gélification; dans le cas où elle serait de nature protidique ce sont les substances attaquant ees matières qui amèneraient la labilisation. En examinant les stabilisants énergiques que nous avons découverts, on y trouve certes des solvants énergiques des graisses, mais aussi des substances sans action sur elles. On aboutit à une impasse.

Toutefois, la couche protectrice pour être attaquée doit être movillable par les substances introduites. En examinant à ce point de vue les corps variés on peut dresser les deux listes suivantes: 
Substances polaires

Ether de pétrole

Chloroforme

Benzène

Toluène

Nitrobenzène
Substances polaires - non polaires

Alcools (éthanol, ete.)

Esters (acétate d'éthyle)

Ce seraient donc les substances apolaires qui auraient un effet stabilisant le plus énergique. Les recherches doivent se poursuivre sur ce plan pour obtenir une conclusion nette.

Résumons-nous. A la lumière des expériences relatées, il s'ensuit :

Le mécanisme de la stabilisation procède de celui concernant la gélification du lait, or, cette dernière nécessite, comme nous l'avons vu, un grossissement préalable et approprié des micelles de la caséine et leur hydratation a maxima. Par conséquent, la stabilisation doit empêcher ce grossissement micellaire et l'hydratation consécutive. Mais les micelles de caséine étant protégées par une couche périmicellaire, cette couche doit être attaquée afin que le grossissement des micelles par coalescence se produise. La stabilisation doit viser les buts suivants :

a) Conserver l'intégralité de la couche périmicellaire ;

b) Empêcher le grossissement micellaire, même si la couche est partiellement attaquée.

Pour atteindre le premier but, on doit, au préalable, fixer la nature de la couche protectrice. Les expériences avec les substances décrites, parmi lesquelles un certain nombre appartient au groupe de solvants des lipides, on peut dire que la couche en question ne semble pas être de nature lipidique.

Le deuxième but peut être atteint en nous basant sur les conditions générales qui régissent la stabilité des colloïdes, c'est-à-dire la tension interfaciale, la viscosité et la charge électrique : la tension interfaciale basse, la viscosité du liquide intermicellaireforte et la charge électrique des micelles très accentuée, c'est la triade de la stabilité colloïdale. La stabilisation doit empêcher la décharge électrique des micelles, elle doit apporter un supplément de charges négatives dans la gélification par les acides d'où la stabilisation par l'addition des bases ou par la présence des tampons appropriés.

Les substances diminuant la tension aux interfaces stabilisent le lait ainsi que nous l'avons vu : on peut dire qu'en dehors d'électrolytes toutes les substances organiques étudiées sont actives à 
ce point de vue; or, elles abaissent, en général très fortement la tension aux interfaces.

Mais il reste la stabilisation par les électrolytes : aussi bien que les anions, eertains cations bivalents stabilisent énergiquement le lait. Dans certains cas leur action coïncide avec leur pouvoir dit "antibiotique" ou "anticatalytique "; dans d'autres cas - non (citrate, acétate, magnésium, fer, calcium, palladium, cuivre, etc.). Mais de toute façon c'est tirer un rideau devant notre intelligence que de se contenter des termes " antisepsie"; "antibiose ", "anticatalyse ", etc.

Néanmoins, c'est dans la voie d'investigations colloïdales que nous pensons pouvoir engager les chercheurs pour fixer un jour le mécanisme de la stabllisation du lait.

\section{E. Considérations pratiques}

De la théorie à la pratique il y a loin dans certains cas. En effet, dans les pages préeédentes nous ne nous sommes pas préoceupé de divers détails d'ordre pratique, dont l'importance est primordiale. Tout d'abord, on doit écarter les produits dont la toxicité est avérée. Certains auteurs, sous prétexte qu'un produit donné est peu ou point toxique se croient autorisés de recommander son emploi dans l'industrie alimentaire ; mais peut-on affirmer d'une série expérimentale, même bien conduite, et suffisamment étendue, à la non-nocivité d'un tel produit à la longue ? Assurément non, et les récentes investigations sur l'importance d'équilibre minérominéral ou minéro-organique pour l'organisme obligent les chercheurs de reviser leurs conceptions. C'est ainsi que nous nous étonnons que les industriels américains incorporent dans la crème des doses minimes de sublimé. Nous avons vu que ce produit permet de maintenir le lait sous forme liquide durant de longs jours en concentration de $8 / 10.000$; la dose thérapeutique pour l'homme est de 10 à $30 \mathrm{mgr}$. par jour à condition qu'elle ne soit pas répétée trop longtemps ; mais on sait que le contact prolongé de la peau avec la solution à $1 \%$ provoque un érythème intense, une stomatite, des coliques et de la diarrhée et même une oligurie albumineủse ou hémorragique.

Il en est de même pour le fluorure de sodium qui stabilise le lait en concentration finale de $3 / 1.000$; mais, cette fois aussi, on sait que l'ingestion de $250 \mathrm{mgr}$. de ce sel provoque chez l'homme des nausées, des vomissements, des diarrhées, etc. ; 1'absorption des doses minimes, durant un temps suffisamment prolongé, produit des altérations dentaires particulières et diminution du poids.

Inutile de multiplier les exemples. On comprend la sage inter- 
diction d'introduire diverses substances dans les aliments conseillée par l'Académie de Médecine en France.

En dehors de la toxicité on doit envisager la facilité avec laquelle on peut éliminer le produit introduit dans du lait. On ne peut donc retenir dans ce cas que les substances volatiles. Trillat a proposé l'aldéhyde formique, G. Bertrand la chloropicrine ; mais la pratique n'a pas confirmé les expériences du laboratoire. Nous avons essayé de stabiliser le lait avec de l'éther acétique qui bout à $77^{\circ} \mathrm{C}$. ; mais, on n'arrive pas à le chasser, même après l'ébullition, durant 10 minutes, du lait ainsi stabilisé.

Le goût du lait ne doit pas être altéré ; or, certaines substances lui communiquent une saveur peu agréable : le fluorure de sodium et même le glycol, dont la saveur en solution aqueuse est plutôt agréable.

L'odeur fait écarter également un nombre de substances qui ne s'évaporent pas à la température de l'ébullition du lait.

La couleur fait éliminer quelques produits intéressants : ainsi le furfurol qui stabilise le lait prend une couleur noirâtre à la longue, outre son odeur et sa saveur particulière.

Enfin, le prix du stabilisant ne peut intervenir dans l'application pratique, étant donné les doses infimes que l'on doit utiliser, comparé avec les pertes que provoque la gélification du lait durant l'époque des chaleurs.

Nous avons réunis diverses substances particulièrement intéressantes en tant que stabilisants du lait, en accompagnant les résultats obtenus des inconvénients que nous avons observés. (Voir tableau LV ci-après).

On constate qu'aucune substances nouvelles que nous venons de découvrir et qui stabilisent le lait, n'est utilisable par suite des phénomènes secondaires, organoleptiques, en dehors de toute toxicité, qu'elles provoquent. Un stabilisant du lait reste donc à découvrir.

\section{F. Conclusions}

10 En compairant leś effets stabilisants des diverses substances dans les trois cas de gélification du lait - par l'acidification microbienne, par l'acidification directe et par la présure - on constate que se sont surtout les bases, les anions (phosphate, ferrocyanure) qui empêchent ces gélifications ; c'est donc une neutralization chimique.

$2^{\circ}$ Cette neutralisation chimique n'explique pas le phénomène en entier : des cations (calcium, magnésium, cuivre, mercure) stabilisent également; bien plus, l'acétone, l'éther de pétrole léger 
TABLEAU LV

PHÉNOMENES SECONDAIRES DUS A LA STABILISATION DU LAIT

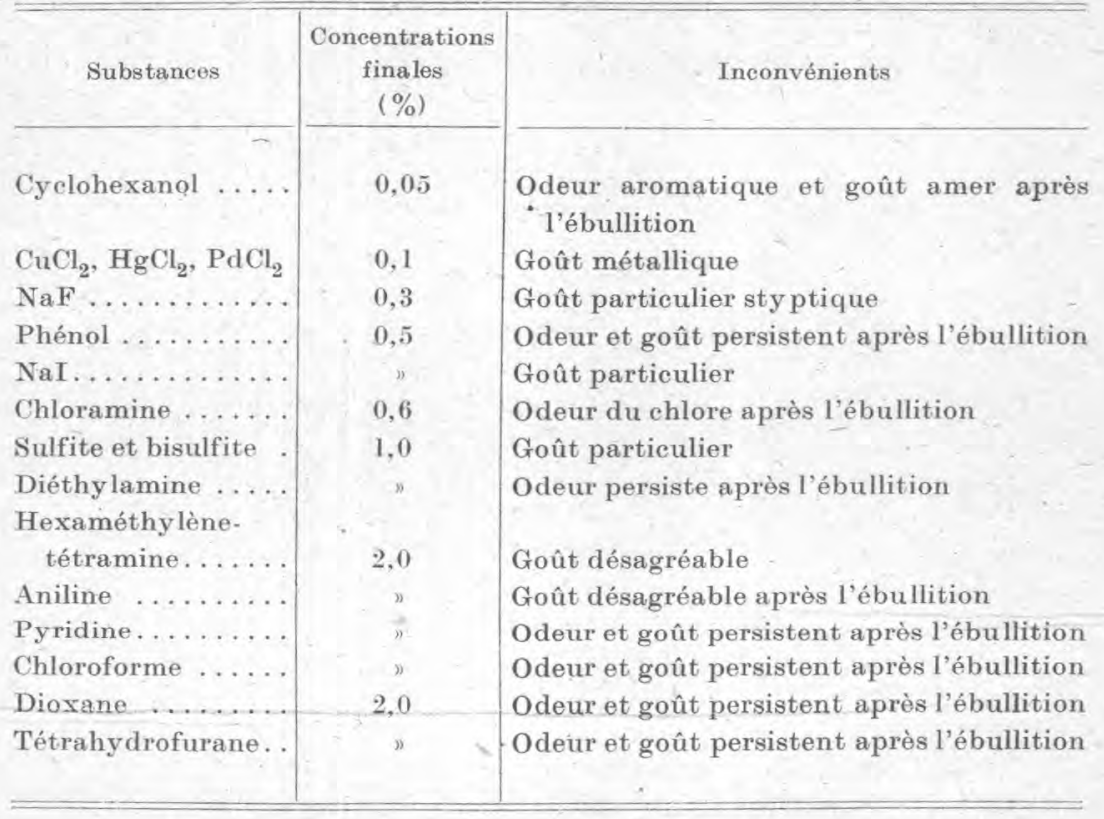

fraction distillant (au-dessous de $55^{\circ}$ C.) et les tétrahydrofurane permettent également d'éviter cette gélification. Les substances organiques en question abaissent très fortement la tension aux interfaces.

$3^{0}$ D'autres substances organiques (éther éthylique ou acétique, dioxane, etc.) également fortement actives au point de vue capillaire, ne permettent que d'éviter une prise en masse, mais une coagulation plus ou moins totale se produit ; dans certains cas, c'est une floculation fine qui a lieu. Dans tous ces cas il y a toujours une séparation du lait en deux phases - la phase liquide étant au fond $\mathrm{du}$ récipient.

$4^{\circ}$ La conservation pratique du lait n'est pas chose aisée : les substances les plus volatiles - éther de pétrole léger, distillant à $55^{\circ}$ C., est pratiquement impossible à éliminer du lait stabilisé, même après une longue ébullition. La recherche d'un stabilisant pratique doit s'inspirer des données concernant les conditions d'équilibre colloïdal.

\section{BIBLIOGRAPHIE}

J. Alexander. Colloid Chemistry, t. I, 1926, p. 622 .

ANoNYMe. Ice Cream Journal, t. XXII, 1943, p. 33 (I).

O. Ballarin. Notes sur la biochimie du lait, Rio de Janeiro, 1947. Mier, édit. 
Von Behring. Zentrbl. Bakteriol., t. III, 1886, p. 27.

Bergey. Manuel of Determination bacteriology Baltimore, 1934. William et Willkin, édit.

G. Bertrand. C. R. Acad. Sc., t. CCXIX, 1944, p. 230 et t. CCXX, 1945, p. 721 .

G. Bertrand. Conservation du lait. Conférence Palais de la Découverte, Paris, 1947.

P. Bert et Regnard. C. R. Acad. Sci., 1882.

L. Blanchard. Le Lait, t. XXVIII, 1948, p. 148.

BLONDEAU. Journal Pharmac. et Chim., t. XII, 1847, p. 224 et 336.

Bredig. Anorganisehe Fermente, Leipzig, 1901.

Browning. Journal Bacteriol. et Pathol., t. XVIII, 1913, p. 144.

H. D. Dakın. Manuel des antiseptiques, Paris, 1918. Vigot, édit.

J. Decaie. Thèse vétér., Lyon, 1944.

Demeter. Deut. Molkerei Zeit., t. XLV, 1941, p. 1052.

Dénier. Rev. de Pathol. comparée, 1937, p. 870.

M. Doladilhe et A. Guy, C. R. Acad. Sc., 1940.

A. Fodor. Ferment problem, Dresden, 1922. Steinkopff, édit.

M. Fovassier. Ann. Falsific., t. XXVI, 1934, p. 24.

Freudereich. Zentrbl. Bakteriol. t. IV, 1898, p. 309.

Gerber. Soc. Biol., t. LXIV, 1907, p. 783.

GILles. Soc. Biol., t. CXXXVIII, 1944, p. 565.

Gorini. C. R. Acad. Sc., t. CCXVI, 1943, p. 783.

Gratz. Le Lait, t. XIV, 1934, p. 195.

K. E. Harshbahrer. Journ. Dairy.Sc., t. XXV, 1942, p. 169 (I).

Hata. Biochem. Zeit, t. XVII, 1909, p. 156.

KaUfmann. Zeit. Physiol. Chem., t. XL, 1903, p. 434.

H. D. KAY, Le Lait, t. XXV, 1945, p. 353.

W. Kopaczewski. Thérapie, t. III, 1948, p. 109.

W. Kopaczew ski. Arch, Mikrobiologie, t. II, 1931, p. 187.

W. KREI. Biedermann's Zentrabl., t. XIII, 1941, p. 211 (I).

Lasabliť̀ Re. Bull. Aead. Méd., t. CXXX, 1946, p. 371.

M. Martel. Bull. Acad. Méd., 22 mai 1945.

A. MAYer. Landw. Versuchst., t. XXVII, 1882, p. 247.

A. Meyer. Gaz de combat, Paris, 1938. Lavauzelle, édit., p. 12.

E. Merck. Merck's Ber., 1908, p. 179.

Miquel. Les organismes vivants de l'atmosphère, Paris, 1883.

A. Monvorsin. Le Lait, Paris, 1925. Vigot, édit.

A. Moret et A. Rochaix. Bull. Sc. Pharmac., t. XXXII, 1925, p. 257.

H. Nitschmann et H. Lauener. Helv. Chim. Acta, t. XXIX, 1946, p. 190.

Pfleiderer. Pflueger's Arch, t. LXVI, 1897, p. 605.

J. Pien. Le Lait, t. XXVIII, 1948, p. 1.

Pien et Baisse. Le Lait, t. XVI, 1936, p. 921.

Ch. Richet, Biochem. Zeit., t. II, 1908, p. 273.

St. Rusznyak. Zeit. physik. Chem., t. LXXXV, 1913, p. 681.

SCHOENDORFF et Victorow. Pflueger's Areh., t. CX, 1907, p. 495.

J. Stilling. Anilinsfarbstoffe als Antiseptika, Marburg, 1890. Teubner, édit. 
M. Tiffeneau. Paris médical, 1930, p. 567.

G. Thiedun, Le Lait, t. XXVIII, 1948, p. 1.

VIElley et HARDer. Le Lait, t. XVII, 1937, p. 707.

Wroblew SKI. Ber. deut, chem. Ges., t. XXVIII, 1895, p. 1719.

M. W. YALE. Le Lait, t. XV, 1935, p. 467.

Young. Cité d'après Tiffenead, loc. cit.

Edg. Zunz. Eléments de Pharmacodynamie, Paris, 1932. Masson, édit., p. 1053 , passim.

\title{
SUR LES STREPTOCOQUES DES MAMIMITES BOVINES (1)
}

\author{
par \\ L. COTONI, P. FORGEOT et G. THIEULIN
}

$\mathrm{Au}$ cours de recherches bactériologiques anciennes sur les streptocoques, nous avions été amenés à étudier les caractères des streptocoques de la mammite contagieuse des vaches laitières. La lecture des travaux étrangers nous faisait souhaiter d'étudier également l'épidémiologie et la prophalaxie de cette affection, d'une importance éeonomique si grande. L'un de nous, en collaboration. avec BEAURRère et-GÉLX, s'est attaché à cette étude, dans le domaine de l'inspection sanitaire, et les résultats en ont été publiés ailleurs. Des difficultés pratiques causées par la guerre ayant ralenti nos recherches, nous rapportons dès maintenant les résultats purement bactériologiques, tirés de l'étude d'un certain nombre d'échantillons de streptocoques.

Dans notre pays, où Nodard et Mollereau ont mis en lumière l'agent pathogène causant de nombreuses mammites (1884), très rares sont les études consacrées à cette affection. Au contraire, à l'étranger, on rencontre des travaux nombreux et étendus. Rappelons entre autres noms, ceux de Minett, Stableforth et EDWARDS, qui ont fait sur les mammites des recherches cliniques, bactériologiques et épidémiologiques particulièrement importantes ; en Allemagne, Klimmer et Haupt, Sielemand ; au Canada, Rosell ; en Autriche, Diernhofer (1936) ; en Suisse, Steck, ZOLLIKOFER, etc.

\section{Isolement des échantillons de streptocoque}

Ces échantillons proviennent de l'ensemencement de, laits obtenus par traite séparée des différents quartiers de la mamelle chez des vaches suspectes ou atteintes de mammite. Les lésions

(1) Annales de l'Institut Pasteur, 1946, 72, 184 (cinq tableaux récapitulatifs, non reproduits ici). 